2016-05

The optimal design of a flap gate array in front of a straight vertical wall: Resonance of the natural modes and enhancement of the exciting torque

Michele, Simone

http://hdl.handle.net/10026.1/17509

10.1016/j.oceaneng.2016.04.002

Ocean Engineering

Elsevier BV

All content in PEARL is protected by copyright law. Author manuscripts are made available in accordance with publisher policies. Please cite only the published version using the details provided on the item record or document. In the absence of an open licence (e.g. Creative Commons), permissions for further reuse of content should be sought from the publisher or author. 


\title{
The optimal design of a flap gate array in front of a straight vertical wall: Resonance of the natural modes and enhancement of the exciting torque
}

\author{
S. Michele*, P. Sammarco, M. d'Errico \\ Department of Civil Engineering and Computer Science, Università degli Studi di Roma “Tor Vergata”, Via del Politecnico 1, 00133 Roma, Italy
}

\section{A R T I C L E I N F O}

\section{Article history:}

Received 16 December 2015

Received in revised form

24 February 2016

Accepted 5 April 2016

Available online 19 April 2016

Keywords:

Wave energy extraction

Wave body interaction

Resonance

\begin{abstract}
A B S T R A C T
We consider a wave energy converter made of an array of $Q$ neighbouring floating flap gates with finite thickness in front of a straight vertical wall in constant depth. Solutions of the radiation and scattering problems are achieved by application of Green's theorem and Green's function yielding a system of hypersingular integral equations for the velocity potential expanded in terms of Legendre polynomials. We investigate how the distance between the array and the vertical wall affects the performance of the array under the action of monochromatic and random waves. We show that large values of the exciting torque on the gates can be obtained by tuning the wall distance with the resonance of the natural modes of the array; this in turn yields large power extraction for a wide range of frequencies.
\end{abstract}

(c) 2016 Elsevier Ltd. All rights reserved.

\section{Introduction}

Research on wave energy converters (WECs) has undergone a remarkable development in recent years. Lots of attention have been given to the individuation of the optimal layout which maximizes power absorption. Lately, the oscillating wave surge converter (OWSC) has been proven very effective (Babarit et al., 2012; Renzi et al., 2014; Whittaker and Folley, 2012). Analytical and numerical models have been developed to analyse the behaviour of a single OWSC in a channel (Renzi and Dias, 2012) and in open sea (Renzi and Dias, 2013). The occurrence of resonant amplification due to interaction of multiple floating bodies through wave motion has been investigated in Adamo and Mei (2005), Li and Mei (2003), Falnes and Hals (2012), Panizzo et al. (2006), Sammarco et al. (1997), Sammarco et al. (1997), Srokosz and Evans (1979), and Thomas and Evans (1979). Consistently, the analysis has been extended to systems of multiple OWSCs in which several piercing gates interact (Michele et al., 2015; Renzi and Dias, 2014; Renzi et al., 2014; Sammarco et al., 2013; Sarkar et al., 2014). Works have focused also on investigating the behaviour of a single wave energy converter near a reflecting vertical wall (Evans, 1988; Evans and Porter, 1995, 1996; Lovas et al., 2010; Martins-Rivas and Mei, 2009; Sarkar et al., 2015). Evans (1988) solved the problem of a point absorber in front of a

\footnotetext{
* Corresponding author.

E-mail addresses: michele@ing.uniroma2.it (S. Michele), sammarco@ing.uniroma2.it (P. Sammarco), derrico@ing.uniroma2.it (M. d'Errico).
}

vertical wall. He found that for certain values of the distance between the wall and the point absorber, the capture width (Mei et al., 2005) reaches large values. Similar results have been obtained by Sarkar et al. (2015) for a flap-type oscillating wave energy converter with small thickness near a straight coast. Evans and Porter (1996) solved the two-dimensional problem of a thin rolling plate next to a vertical wall. They found that strong resonance occurs at the frequencies of the sloshing modes. However the effects of a straight vertical wall on the behaviour of a system of OWSC's have not been investigated yet.

In this paper, the behaviour of a single array made of $Q$ floating flap gates with finite thickness in front of a straight vertical wall is analysed. We extend the solution of Michele et al. (2015) for the radiation and scattering problems in terms of Green's function and hypersingular integral equations. In order to account for the noflux boundary condition at the wall, the Green function has been modified by making use of the method of images (Linton and Mclver, 2001; Morse and Feshbach, 1981). The complexity of the boundaries is then reduced and the mathematical problem considerably simplified. We show that the array in front of a reflecting wall achieves larger values of the capture factor with respect to both the case of a gate farm in open sea (Michele et al., 2015) and a single gate in front of a reflecting wall (Sarkar et al., 2015). Indeed, such a system benefits from the mutual interaction between the resonance of the natural modes and from the wall induced enhancement of the exciting torque. A parametric analysis in monochromatic incident waves reveals also to what extent the distance between array and vertical wall can affect the efficiency of the device. Finally, the behaviour and performance of the array 
under the action of incident waves represented by the JONSWAP spectrum is investigated and discussed (Hasselmann et al., 1973; Goda, 2012; Eriksson et al., 2005; Sarkar et al., 2013).

\section{Governing equations for $Q$ gates}

Referring to Fig. 1, consider a finite single array made of $Q$ identical flap gates in front of a reflecting vertical wall. Let $h$ be the constant water depth and $L$ the distance between the array and the wall. $a$ and $2 b$ are respectively the width and the thickness of each gate, hence $w=a Q$ represents the total width of the array. Define a three-dimensional Cartesian coordinate system with the $x$ and $y$ axes lying on the mean free surface and the $z$ axis pointing vertically upward. The $y$ axis is directed along the wall which spans from $y=-\infty$ to $y=\infty$, while the $x$ axis is orthogonal both to the array and to the vertical wall. All the gates are hinged to a bottom foundation and oscillate about the horizontal common axis lying on $x=L, z=-h+c$. The symbol $G_{q}$ denotes the $q$-th flap gate. Monochromatic waves of amplitude $A$, period $T$ and angular frequency $\omega=2 \pi / T$ come from $x=+\infty$ and are normally incident on the flaps. Let $\Theta_{q}(t)$ be the angular displacement of $G_{q}$, positive clockwise and define $\Theta(y, t)$ as the angular displacement function of the array:

$\Theta(y, t)=\left\{\Theta_{1}(t), \ldots, \Theta_{q}(t), \ldots, \Theta_{Q}(t)\right\}$.

$\Theta(y, t)$ is an unknown piece-wise function of $y$. The fluid is considered inviscid and incompressible and the flow irrotational, hence there exist a velocity potential $\Phi(x, y, z, t)$ satisfying the Laplace equation in the fluid domain $\Omega$ :

$\nabla^{2} \Phi=0, \quad(x, y, z) \in \Omega$.

On the basis of linearised water-wave theory the potential $\Phi(x, y, z, t)$ satisfies

$\frac{\partial^{2} \Phi}{\partial t^{2}}+g \frac{\partial \Phi}{\partial z}=0, \quad z=0$,

and
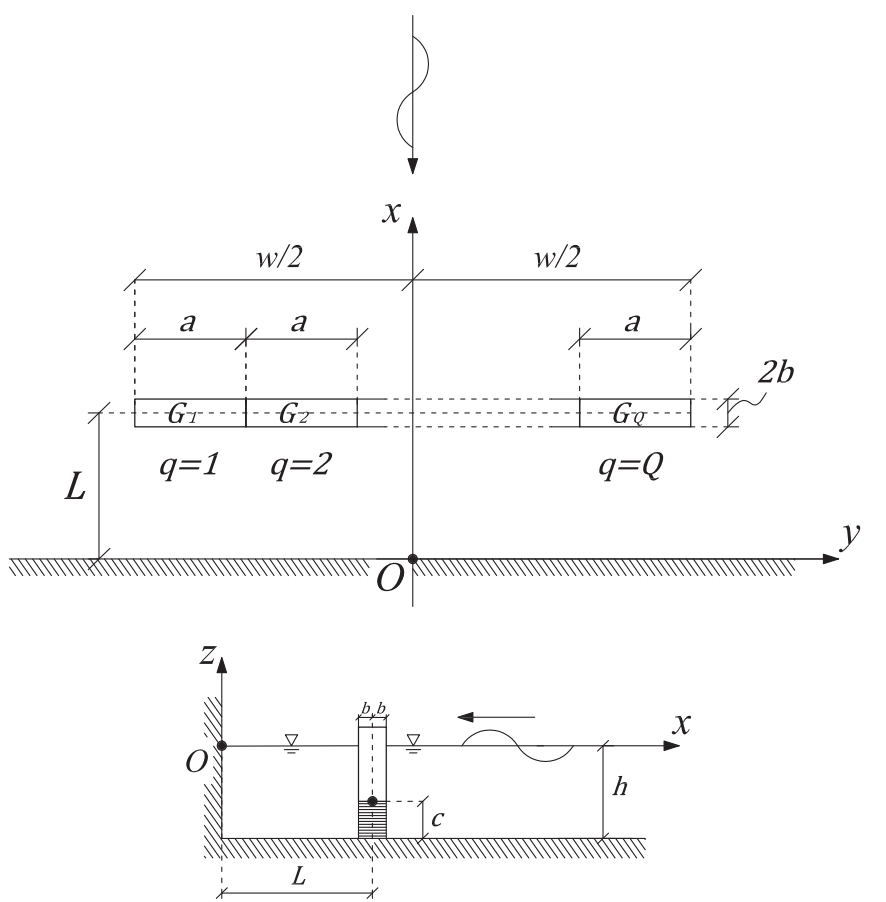

Fig. 1. Plan geometry and side view. $\frac{\partial \Phi}{\partial z}=0, \quad z=-h$.

In the limit of small-amplitude oscillations, the kinematic condition on the array may be written:

$\frac{\partial \Phi}{\partial x}=\frac{d \Theta_{p}}{d t}(z+h-c) H(z+h-c)$,

$x=L \pm b, \quad y \in\left(-\frac{w}{2}, \frac{w}{2}\right), \quad z \in[-h, 0]$,

$\frac{\partial \Phi}{\partial y}=0, \quad x \in(L-b, L+b), \quad y= \pm \frac{w}{2}, \quad z \in[-h, 0]$.

where $H$ is the Heaviside step function, while the kinematic condition on the vertical wall gives:

$\frac{\partial \Phi}{\partial x}=0, \quad x=0, \quad z \in[-h, 0]$.

Assuming harmonic fluid and gates motion with frequency $\omega$, the time dependence can be separated as follows:

$\Phi(x, y, z, t)=\operatorname{Re}\left\{\phi(x, y, z) e^{-\mathrm{i} \omega t}\right\}$,

$\Theta_{q}(y, t)=\operatorname{Re}\left\{\theta_{q}(y) e^{-\mathrm{i} \omega t}\right\}$.

The problem is linear, hence the spatial potential $\phi(x, y, z)$ can be decomposed as:

$\phi=\phi^{I}+\phi^{F}+\phi^{S}+\sum_{q=1}^{Q} \phi_{q}^{R}$,

where:

$\phi^{I}=-\frac{\mathrm{i} A g}{\omega} \frac{\operatorname{ch~} k_{0}(h+z)}{\operatorname{ch} k_{0} h} e^{-\mathrm{i} k_{0} x}$,

is the potential of the plane incident waves incoming from $x=+\infty$,

$\phi^{F}=-\frac{\mathrm{i} A g}{\omega} \frac{\mathrm{ch} k_{0}(h+z)}{\operatorname{ch} k_{0} h} e^{\mathrm{i} k_{0} x}$,

is the potential of the waves reflected by the vertical wall, $\phi^{S}$ is the potential of the scattered waves by the array and $\phi_{q}{ }^{R}$ is the potential of the radiated waves due to the moving gate $G_{q}$ while all the other gates are at rest. In (11) and (12) $k_{0}$ is the wavenumber, i.e. the real root of the dispersion relation $\omega^{2}=g k_{0}$ th $k_{0} h$ and $i$ is the imaginary unit. Both $\phi_{q}{ }^{R}$ and $\phi^{S}$ must satisfy Laplace equation (2), the mixed boundary condition on the free surface (3) the no-flux condition on the seabed (4) and the no-flux condition on the vertical wall (7).

Let $x^{ \pm}$indicate the $x$-coordinate of the rest position of the vertical surface of the gates:

$x^{ \pm}=L \pm b$.

Each gate $G_{q}$ spans a $y$-width given by:

$y \in\left[y_{q}, y_{q+1}\right], \quad y_{q}=(q-1) a-\frac{w}{2}, \quad q=1, \ldots, Q$.

Define the horizontal boundary $S_{q}$ of the gate $G_{q}$ as

$S_{q}=\left\{x=x^{ \pm}, y \in\left[y_{q}, y_{q+1}\right]\right\}$,

and the end horizontal boundaries of the array

$S_{w}=\left\{x \in\left(x^{-}, x^{+}\right), y= \pm \frac{w}{2}\right\}$.

The kinematic boundary conditions on the array surfaces and on the vertical wall can be written as follows: 
$\frac{\partial \phi_{q}^{R}}{\partial x}= \begin{cases}-\mathrm{i} \omega \theta_{q}(z+h-c) H(z+h & x, y \in S_{q}, z \in[-h, 0], \\ -c), & \text { elsewhere on the array, } \\ 0 & x=0, z \in[-h, 0]\end{cases}$

$\frac{\partial \phi_{q}^{R}}{\partial y}=0, \quad x, y \in S_{w}, z \in[-h, 0]$,

$\frac{\partial \phi^{S}}{\partial x}= \begin{cases}-\frac{\partial \phi^{I}}{\partial x}-\frac{\partial \phi^{F}}{\partial x}, & x, y \in S_{q}, \quad z \in[-h, 0], \\ 0, & x=0, z \in[-h, 0]\end{cases}$

$\frac{\partial \phi^{S}}{\partial y}=0, \quad x, y \in S_{w}, z \in[-h, 0]$

Finally $\phi^{(R, S)}$ must be bounded at large distances from the array, i.e. for $\sqrt{x^{2}+y^{2}} \rightarrow \infty$.

\section{Solution of the scattering and radiation potentials}

In order to find $\phi^{S}$ and the $\phi_{q}{ }^{R^{\prime}}$ S we first separate the vertical dependence:

$\left.\begin{array}{l}\phi_{q}^{R} \\ \phi^{S}\end{array}\right\}=\sum_{n=0}^{\infty} Z_{n}(z)\left\{\begin{array}{l}\varphi_{n, q}^{R}(x, y) \\ \varphi_{n}^{S}(x, y)\end{array}\right.$,

where $Z_{n}(z)$ are the normalized eigenfunctions:

$Z_{n}(z)=\frac{\sqrt{2} \operatorname{ch} k_{n}(h+z)}{\left(h+\frac{g}{\omega^{2}} \operatorname{sh}^{2} k_{n} h\right)^{1 / 2}}, \quad \int_{-h}^{0} Z_{n} Z_{m} d z=\delta_{n m}$,

$n, m=0,1, \ldots$

and $\delta_{n m}$ is the Kronecker delta. In (22), $k_{n}$ for $n \geq 1$ are the complex roots of the dispersion relation:

$k_{n}=\mathrm{i} \bar{k}_{n}, \quad \omega^{2}=-g \bar{k}_{n} \tan \bar{k}_{n} h, \quad n=1, \ldots, \infty$.

Following separation (21) the Laplace equation (2) becomes the Helmholtz equation

$\left(\frac{\partial^{2}}{\partial x^{2}}+\frac{\partial^{2}}{\partial y^{2}}+k_{n}^{2}\right)\left\{\begin{array}{c}\varphi_{n, q}^{R}(x, y) \\ \varphi_{n}^{S}(x, y)\end{array}\right\}=0$.

The boundary conditions (17)-(20) then become

$\frac{\partial \varphi_{n, q}^{R}}{\partial x}= \begin{cases}-\mathrm{i} \omega \theta_{q} f_{n} & \text { on } S_{q}, \\ 0 & \text { elsewhere on the array, } \\ 0, & x=0,\end{cases}$

$\frac{\partial \varphi_{n, q}^{R}}{\partial y}=0, \quad$ on $S_{w}$

$\frac{\partial \varphi_{n}^{S}}{\partial x}= \begin{cases}A d_{n} \sin k_{n} x & \text { on } S_{q}, \\ 0, & x=0,\end{cases}$ $\frac{\partial \varphi_{n}^{S}}{\partial y}=0$ on $S_{w}$

$q=1, \ldots, Q$.

Finally, $\varphi_{n, q}^{R}$ and $\varphi_{n}{ }^{S}$ must be bounded for $\sqrt{x^{2}+y^{2}} \rightarrow \infty$. In (25)(28), the coefficients $f_{n}$ and $d_{n}$ are given by

$$
\begin{aligned}
& f_{n}=\int_{-h}^{0} Z_{n}(z+h-c) H(z+h-c) d z \\
& =\frac{\sqrt{2}\left[\operatorname{ch} k_{n} c-\operatorname{ch} k_{n} h+k_{n}(h-c) \operatorname{sh} k_{n} h\right]}{\left(h+\frac{g}{\omega^{2}} \operatorname{sh}^{2} k_{n} h\right)^{1 / 2} k_{n}^{2}}, \quad n=0,1, \ldots
\end{aligned}
$$

$$
\begin{aligned}
d_{0} & =-\frac{2 \operatorname{ig} k_{0}}{\omega \operatorname{ch} k_{0} h} \int_{-h}^{0} Z_{0} \operatorname{ch} k_{0}(h+z) d z \\
& =-\frac{\sqrt{2} \operatorname{ig} k_{0}\left(h+\frac{g}{\omega^{2}} \operatorname{sh}^{2} k_{0} h\right)^{1 / 2}}{\omega \operatorname{ch} k_{0} h}
\end{aligned}
$$

$d_{n}=0 n=1,2, \ldots$

We employ the method of Michele et al. (2015) properly modified to account for the vertical wall. The solution is found via the method of integral equations based on Green's theorem and Green's function.

Consider the plane fluid domain $\Sigma$ enclosed within the boundary of the array, the vertical wall and a semi-circle of large radius $S_{\infty}$ surrounding the array. By using the method of images, the Green's function for the semi infinite domain $\Sigma$ is given by:

$G_{n}(x, y ; \xi, \eta)=-\frac{\mathrm{i}}{4}\left[G_{n}^{(0)}(x, y ; \xi, \eta)+G_{n}^{(1)}(x, y ; \xi, \eta)\right]$,

where

$G_{n}^{(0)}(x, y ; \xi, \eta)=H_{0}^{(1)}\left[k_{n} \sqrt{(x-\xi)^{2}+(y-\eta)^{2}}\right]$,

and

$G_{n}^{(1)}(x, y ; \xi, \eta)=H_{0}^{(1)}\left[k_{n} \sqrt{(x+\xi)^{2}+(y-\eta)^{2}}\right]$,

in which $H_{0}^{(1)}$ is the Hankel function of the first kind and order zero. $G_{n}^{(1)}$ accounts for the reflection due to the vertical wall at $x=0$. Application of the Green's theorem, yields:

$\frac{1}{2}\left\{\begin{array}{c}\varphi_{n, q}^{R}(x, y) \\ \varphi_{n}^{S}(x, y)\end{array}\right\}=\int_{S_{A}}\left[\left\{\begin{array}{c}\varphi_{n, q}^{R}(\xi, \eta) \\ \varphi_{n}^{S}(\xi, \eta)\end{array}\right\} \frac{\partial G_{n}}{\partial n_{p}}-G_{n} \frac{\partial}{\partial n_{p}}\left\{\begin{array}{c}\varphi_{n, q}^{R}(\xi, \eta) \\ \varphi_{n}^{S}(\xi, \eta)\end{array}\right.\right.$

$(x, y) \in S_{A}$,

where $S_{A}=\sum_{q=1}^{Q} S_{q} \cup S_{w}$ is the entire array boundary; the subscripts on the normal coordinate $n_{p}$ and the line element $d S_{p}$ indicate that these are associated with the source point $p=(\xi, \eta) \in S_{A}$ (see also Linton and Mclver, 2001).

Let $\xi^{ \pm}$and $\eta_{q}$ be:

$\xi^{ \pm}=x^{ \pm}, \quad \eta_{q}=y_{q}$.

The boundary of $S_{A}$ is rectangular, hence the normal derivative inside (35) becomes: 
$\frac{\partial}{\partial n_{p}}=\left\{\begin{array}{ll}\mp \frac{\partial}{\partial \xi} & \text { on } S_{q} \\ \mp \frac{\partial}{\partial \eta} & \text { on } S_{w}\end{array}\right.$.

Substitution of the boundary conditions (25)-(28) inside (35), yields:

$$
\begin{aligned}
\frac{1}{2} \varphi_{n, q}^{R}(x, y)= & -\int_{-\frac{w}{2}}^{\frac{w}{2}}\left(\left.\varphi_{n, q}^{R} \frac{\partial G_{n}}{\partial \xi}\right|_{\xi=\xi^{+}}-\left.\varphi_{n, q}^{R} \frac{\partial G_{n}}{\partial \xi}\right|_{\xi=\xi^{-}}\right) d \eta \\
& -\int_{\xi^{-}}^{\xi^{+}}\left(\left.\varphi_{n, q}^{R} \frac{\partial G_{n}}{\partial \eta}\right|_{\eta=\frac{w}{2}}-\left.\varphi_{n, q}^{R} \frac{\partial G_{n}}{\partial \eta}\right|_{\eta=-\frac{w}{2}}\right) d \xi \\
& +\mathrm{i} \omega \theta_{q} f_{n} \int_{\eta_{q}}^{\eta} \eta_{q+1}\left(\left.G_{n}\right|_{\xi=\xi^{-}}-\left.G_{n}\right|_{\xi=\xi^{+}}\right) d \eta,(x, y) \in S_{A}, \\
\frac{1}{2} \varphi_{n}^{S}(x, y)= & -\int_{-\frac{w}{2}}^{\frac{w}{2}}\left(\left.\varphi_{n}^{S} \frac{\partial G_{n}}{\partial \xi}\right|_{\xi=\xi^{+}}-\left.\varphi_{n}^{S} \frac{\partial G_{n}}{\partial \xi}\right|_{\xi=\xi^{-}}\right) d \eta \\
& -\int_{\xi^{-}}^{\xi^{+}}\left(\left.\varphi_{n}^{S} \frac{\partial G_{n}}{\partial \eta}\right|_{\eta=\frac{w}{2}}-\left.\varphi_{n}^{S} \frac{\partial G_{n}}{\partial \eta}\right|_{\eta=-\frac{w}{2}}\right) d \xi \\
& +A d_{n} \int_{-\frac{w}{2}}^{\frac{w}{2}}\left(\left.\sin \left(k_{n} \xi\right) G_{n}\right|_{\xi=\xi^{+}}-\sin \left(k_{n} \xi\right) G_{n} \mid \xi=\xi^{-}\right) d \eta,(x, y) \in S_{A},
\end{aligned}
$$

which are similar to the radiation and scattering potentials obtained in Michele et al. (2015) but with a different Green's function. The solution of the radiation and scattering problems is achieved once the boundary conditions (25)-(28) on the array are enforced. The resulting system of hypersingular integral equations is finally solved by expanding $\varphi_{n, q}^{R}$ and $\varphi_{n}^{S}$ in terms of Legendre polynomials $P_{m}$ of integer order $m=0, \ldots, M$ and by usage of the collocation scheme based on the zeros of the first-kind Chebyshev polynomials (see the Appendix for further details). Finally, the radiation and the scattering potentials on the boundary of the array are given by:

$\left.\begin{array}{l}\phi_{q}^{R}\left(x^{ \pm}, y, z\right) \\ \phi_{q}^{R}\left(x, \pm \frac{w}{2}, z\right)\end{array}\right\}=\sum_{n=0}^{\infty} \sum_{m=0}^{M} Z_{n}(z) \theta_{q}\left\{\begin{array}{l}P_{m}(y) \alpha_{n m, q}^{R_{ \pm}}, \\ P_{m}(x) \beta_{n m, q}^{R+},\end{array}\right.$

$\left.\begin{array}{l}\phi^{S}\left(x^{ \pm}, y, z\right) \\ \phi^{S}\left(x, \pm \frac{w}{2}, z\right)\end{array}\right\}=\sum_{m=0}^{M} Z_{0}(z)\left\{\begin{array}{l}P_{m}(y) \alpha_{0 m}^{S_{ \pm}} \\ P_{m}(x) \beta_{0 m}^{S_{ \pm}}\end{array}\right.$,

$x \in(-b+L, b+L), \quad y \in\left(-\frac{w}{2}, \frac{w}{2}\right)$,

where $\alpha_{n m, q}^{R_{ \pm}}, \alpha_{0 m}^{S_{ \pm}}, \beta_{n m, q}^{R_{ \pm}}$and $\beta_{0 m}^{S_{ \pm}}$are complex constants determined by solving the linear systems (A.30a)-(A.30c) and (A.31a)-(A.31b) (see the Appendix), while the \pm symbol above $\alpha$ and $\beta$ refers respectively to $x^{ \pm}$and $\pm \frac{w}{2}$.

\section{Gate motion and hydrodynamic parameters}

Consider each gate $G_{q}$ coupled with an energy generator at the hinge and assume that the generator exerts a torque proportional to the angular velocity of the gate $G_{q}$. The angular momentum equation of the flap is that of a damped harmonic oscillator:

$$
\begin{aligned}
& I \frac{d^{2} \Theta_{q}}{d t^{2}}+\nu_{p t o} \frac{d \Theta_{q}}{d t}+C \Theta_{q} \\
& \quad=\rho \int_{y_{q}}^{y_{q+1}} d y \int_{-h+c}^{0} \frac{\partial\left(\left.\Phi\right|_{x=x^{+}}-\left.\Phi\right|_{x=x^{-}}\right)}{\partial t}(z+h-c) d z
\end{aligned}
$$

in which $\nu_{\text {pto }}$ is the power take-off coefficient, $I$ is the moment of inertia of the gate about the hinge and $C$ is the net restoring torque:

$C=\rho g\left(I_{x x}^{A}+I_{z}^{V}\right)-M_{g} g\left(z_{g}+h-c\right)$,

where:

$I_{x x}^{A}=\iint_{S_{G}} x^{2} d x d y, \quad I_{z}^{V}=\iiint_{V}(z+h-c) d V$,

$S_{G}$ denotes the cross sectional area of the gate at the water line and $V$ the water volume displaced by the gate in its rest vertical position. $M_{g}$ and $z_{g}$ are respectively the mass and the vertical coordinate of the center of mass of the gate. For the geometry of Fig. $1, \mathrm{I}_{x x}{ }^{A}$ and $\mathrm{I}_{z}{ }^{V}$ are:

$I_{x x}^{A}=\frac{2 a b^{3}}{3}, \quad I_{z}^{V}=a b(h-c)^{2}$.

Using (8)-(10) and the expressions of the incident (11), radiation (40) and scattering (41) potentials, the angular momentum equation (42) becomes

$$
\begin{gathered}
\left(-\omega^{2} I+C-\mathrm{i} \omega \nu_{p t o}\right) \theta_{q}-\sum_{p=1}^{Q} \theta_{p}\left(\omega^{2} \mu_{p}^{q}+\mathrm{i} \omega \nu_{p}^{q}\right)=F_{q}, \\
q=1, \ldots Q,
\end{gathered}
$$

where

$$
\begin{aligned}
F_{q}= & -\mathrm{i} \omega \rho\left\{\frac{4 \mathrm{i} A g a \sin k_{0} b \sin k_{0} L\left[\mathrm{ch} k_{0} c-\mathrm{ch} k_{0} h+k_{0}(h-c) \mathrm{sh} k_{0} h\right]}{\omega k_{0}^{2} \mathrm{ch} k_{0} h}\right. \\
& \left.+f_{0} \int_{y_{q}}^{y_{q+1}} \sum_{m=0}^{\infty}\left(\alpha_{0 m}^{S_{+}}-\alpha_{0 m}^{S_{-}}\right) P_{m}\left(\frac{2 y}{w}\right) d y\right\},
\end{aligned}
$$

is the exciting torque due to diffracted waves, while:

$\mu_{p}^{q}=\frac{\rho}{\omega} \operatorname{Im}\left\{\sum_{n=0}^{\infty} f_{n} \int_{y_{q}}^{y_{q+1}} \sum_{m=0}^{M}\left(\alpha_{n m, p}^{R+}-\alpha_{n m, p}^{R-}\right) P_{m}\left(\frac{2 y}{w}\right) d y\right\}$,

and

$\nu_{p}^{q}=-\rho \operatorname{Re}\left\{\sum_{n=0}^{\infty} f_{n} \int_{y_{q}}^{y_{q+1}} \sum_{m=0}^{M}\left(\alpha_{n m, p}^{R+}-\alpha_{n m, p}^{R-}\right) P_{m}\left(\frac{2 y}{w}\right) d y\right\}$,

represent, respectively, the added inertia and the radiation damping of the gate $G_{q}$ due to the unit rotation of the gate $G_{p}$. The matrix form of Eq. (46) is:

$\left[\left(-\omega^{2} I+C-\mathrm{i} \nu_{p t o}\right) \mathbf{I}-\omega^{2} \mathbf{M}-\mathrm{i} \omega \mathbf{N}\right]\{\theta\}=\{F\}$,

where $\{\theta\}$ is a column vector of length $Q$ that contains all the angular displacements of the gates, $\mathbf{I}$ is the identity matrix of size $Q \times Q, \mathbf{M}$ and $\mathbf{N}$ are respectively the added inertia matrix and the radiation damping matrix also of size $Q \times Q$ :

$\mathbf{M}=\left[\begin{array}{ccc}\mu_{1}^{1} & \ldots & \mu_{Q}^{1} \\ \vdots & \ddots & \vdots \\ \mu_{1}^{Q} & \ldots & \mu_{Q}^{Q}\end{array}\right], \quad \mathbf{N}=\left[\begin{array}{ccc}\nu_{1}^{1} & \ldots & \nu_{Q}^{1} \\ \vdots & \ddots & \vdots \\ \nu_{1}^{Q} & \ldots & \nu_{Q}^{Q}\end{array}\right]$,

while $\{F\}$ is a column vector of size $Q$ which contains the values of exciting torque on each gate $G_{q}$ : 
$\{F\}=\left\{\begin{array}{c}F_{1} \\ \vdots \\ F_{q} \\ \vdots \\ F_{Q}\end{array}\right\}$.

Solution of the linear system (50) yields $\{\theta\}$ and the average power absorbed over a wave cycle by the array:

$P=\frac{1}{T} \int_{0}^{T} \nu_{\text {pto }} \sum_{q=1}^{Q}\left(\frac{d \Theta_{q}}{d t}\right)^{2}=\frac{\omega^{2} \nu_{p t o}}{2} \sum_{q=1}^{Q}\left|\theta_{q}\right|^{2}$

Modal analysis can now be performed. The natural modes of the array are the configurations of free undamped motion (Michele et al., 2015; Renzi and Dias, 2014; Sammarco et al., 2013). In order to find the eigenfrequencies and the eigenvectors of the system (50), the damping terms and the exciting torque are set equal to zero and

$\left[\left(-\omega^{2} I+C\right) \mathbf{I}-\omega^{2} \mathbf{M}(\omega)\right]\{\theta\}=0$.

To find non-trivial solutions of (54) the following implicit non linear eigenvalue condition must then be solved:

$\operatorname{det}\left[\left(-\omega^{2} I+C\right) \mathbf{I}-\omega^{2} \mathbf{M}(\omega)\right]=0$.

Once the eigenfrequencies are known, the respective modal forms can be obtained by letting the displacement of the gate $G_{1}=1$ and then solving system (54).

\section{Results and discussion}

\subsection{Validation}

In this section we validate the mathematical model with the results obtained by Sarkar et al. (2015) for a single "thin-gate" hinged on the bottom foundations. Gate and sea characteristics are $w=26 \mathrm{~m}, \quad L=50 \mathrm{~m}, \quad c=4 \mathrm{~m}, \quad h=13 \mathrm{~m}$ and $A=1 \mathrm{~m}$. Assuming $b=0.2 \mathrm{~m}(\ll w)$ we simulate the negligible gate thickness of Sarkar et al. (2015). Fig. 2 shows the variation of added inertia $\mu$, radiation damping $\nu$ and magnitude of the exciting torque $|F|$ versus the dimensionless parameter $k_{0} L$. An excellent agreement can be observed in the results obtained by both models.

5.2. The case of one gate $Q=1$ : the effects of the gate thickness $2 b$ and the distance $L$

The effects of the distance $L$ and the gate thickness $2 b$ are evaluated for the simplest case $Q=1$. Foundation height, water depth, inertia, buoyancy and width of the gate are listed in Table 1. Two values of the distance $L$ and two values of $2 b$ have been chosen, i.e. $L=[1.5 ; 6] \mathrm{m}$ and $2 b=[0.25 ; 1.5] \mathrm{m}$. Fig. 3 shows the dependence of the added inertia $\mu$, the radiation damping $\nu$ and the exciting torque $|F|$ on the incident wave frequency $\omega$.

The effects of the gate thickness become important if the gate is located near the vertical wall, i.e. when $2 b$ is of the same order of magnitude of $L$. It follows that in this case the 'thin-gate' hypothesis is not appropriate.

Consider the case for $L=1.5 \mathrm{~m}, 2 b=1.5 \mathrm{~m}$ : the added inertia is negative for $\omega \in[2.5 ; 3.5] \mathrm{rad} \mathrm{s}^{-1}$, thus the time averaged potential energy exceeds the time averaged kinetic energy, so that freesurface effects are dominant (see Linton and McIver, 2001; Mclver and Evans, 1984 for further details).

Now consider the behaviour of the exciting torque: Fig. 3 (c) exhibits local maxima alternating with values of $|F|$ near zero; the lower the distance $L$ from the wall the larger the maximum

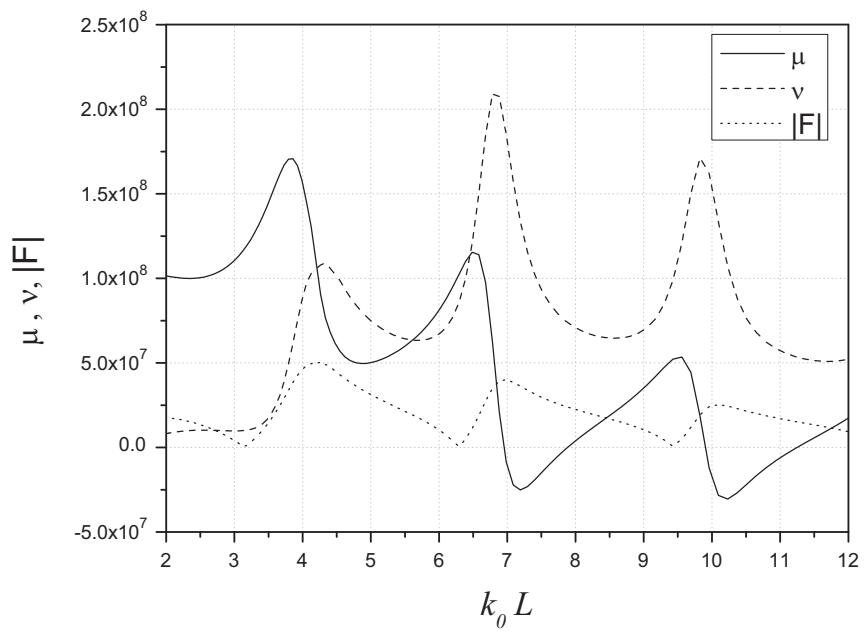

Fig. 2. Added inertia $\mu$, radiation damping $\nu$ and magnitude of the exciting torque $|F|$ versus $k_{0} L$ for $w=26 \mathrm{~m}, L=50 \mathrm{~m}, h=13 \mathrm{~m}$ and $c=4 \mathrm{~m}$. The results are in agreement with those obtained by Sarkar et al. (2015) (see Figs. 2 and 4).

Table 1

Gate characteristics.

\begin{tabular}{lll}
\hline Parameters & Symbol & Value \\
\hline Gate width & $a$ & $3 \mathrm{~m}$ \\
Moment of inertia & $I$ & $72000 \mathrm{~kg} \mathrm{~m}^{2}$ \\
Buoyancy restoring torque & $C$ & $300000 \mathrm{~kg} \mathrm{~m}^{2} \mathrm{~s}^{-2}$ \\
Gate mass & $M_{g}$ & $2600 \mathrm{~kg}$ \\
Foundation height & $c$ & $0 \mathrm{~m}$ \\
Water depth & $h$ & $5 \mathrm{~m}$ \\
Density of water & $\rho$ & $1000 \mathrm{~kg} \mathrm{~m}^{-3}$ \\
\hline
\end{tabular}

value of $|F|$. Similar results have also been found by Sarkar et al. (2015) in the analysis of a 'thin-gate' in front of a straight coast. Such behaviour can be explained considering the interaction of the incident and the reflected wave from the wall which leads to the formation of a standing wave. The locations of the minimum and maximum values correspond respectively to the antinodes and the nodes of the standing wave field. The maximum values of the exciting torque are more than twice that in the open ocean (see Michele et al., 2015). Note also that differently from the case of Sarkar et al. (2015), minima of $|F|$ differ from zero because of the influence of the gate thickness $2 b$.

The behaviour of the radiation damping $\nu$ is similar to $|F|$, i.e. maxima and minima occur at the same frequencies. This phenomena is consistent with the three dimensional Haskind relation, which shows that the exciting torque is proportional to the amplitude of the radiated waves in the far field (Mei et al., 2005)

Let us consider the range of $\omega<2 \mathrm{rad} \mathrm{s}^{-1}$; for given $b$, Fig. 3 (a) shows that the larger the distance $L$ the smaller the added mass $\mu$. As a consequence the first eigenfrequency of the system increases if the distance $L$ increases. On the other hand, if the gate thickness $2 b$ increases also the added mass increases, hence the eigenfrequency must decrease. These trends are reported in Table 2 where first eigenfrequency $\omega_{1}$ of the single gate for the different values of $L$ and $2 b$ is listed.

In this work we consider a perfectly reflecting wall. However, if there is absorption the amplitude of the stationary wave is no longer equal to $2 A \cos 2 k x$. In this case a wave envelope $\tilde{A}=A \sqrt{1+2 R \cos 2 k x+R^{2}}$ which depends on the reflection coefficient $R$ occurs. Since $R<1$, a non-perfect reflecting wall reduces the amplitude of the partial standing wave $\tilde{A}$ and so the exciting torque. Because of the zeros of $\tilde{A}$, also the position of the nodes 
a

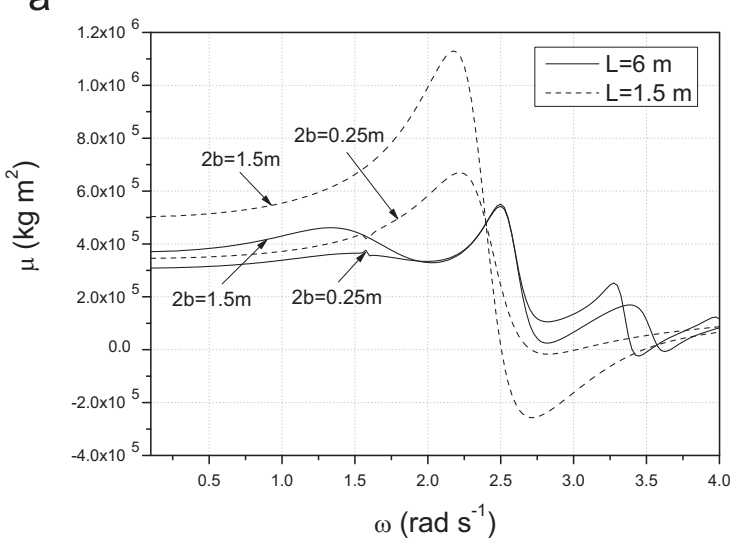

b

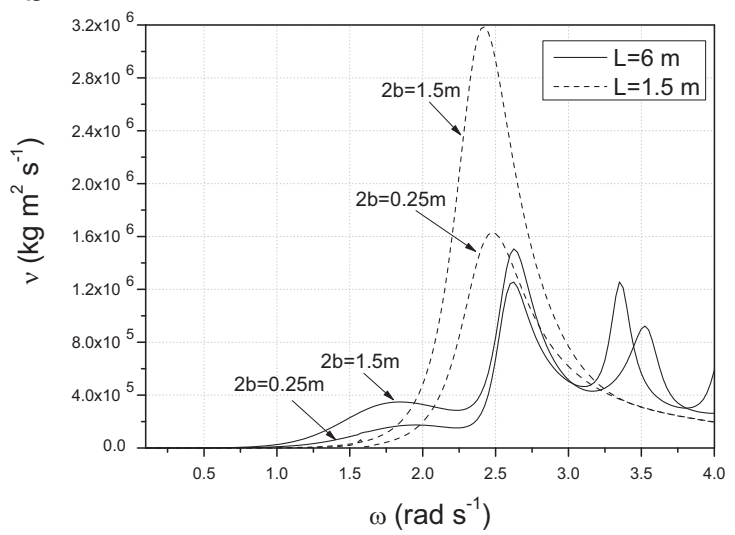

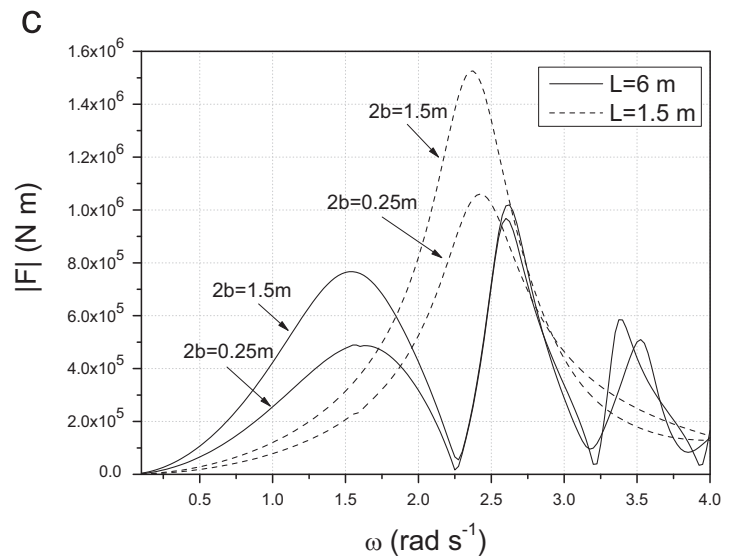

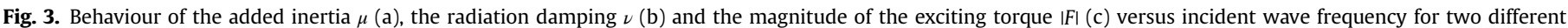
values of the distance $L$ and two different values of the gate thickness $2 b$.

Table 2

Eigenfrequency $\omega_{1}$ of the single gate in the open sea for different values of $L$ and $2 b$. The larger the distance $L$, the larger the eigenfrequency. Conversely, the larger the gate thickness $2 b$, the lower the eigenfrequency.

\begin{tabular}{llll}
\hline$L(\mathrm{~m})$ & $2 b(\mathrm{~m})$ & $\omega_{1}(\mathrm{rad} / \mathrm{s})$ & Period $(\mathrm{s})$ \\
\hline 1.5 & 1.5 & 0.7 & 8.97 \\
1.5 & 0.25 & 0.83 & 7.56 \\
6 & 1.5 & 0.8 & 7.85 \\
6 & 0.25 & 0.86 & 7.30 \\
\hline
\end{tabular}

and antinodes depends on $R$ and the location of maxima and minima of $|F|$ changes in the frequency domain.

\subsection{The case of multiple gates in an array}

Referring to Fig. 1, consider now an array with $Q=7$ gates. Different values of $L$ are chosen, i.e. $L \in[2 ; 60] \mathrm{m}$, the gate thickness is $2 b=1.5 \mathrm{~m}$, while the numerical values of the other parameters are listed in Table 1.

\subsubsection{Eigenfrequencies and eigenvectors}

Solution of (55) gives the numerical values of the eigenfrequencies of the system while the corresponding modal forms are obtained by (54).

Consider a range of $\omega$ from 0.1 to $1.4 \mathrm{rad} \mathrm{s}^{-1}$; the frequency range includes the $Q-1$ natural frequencies of the out-of-phase modes and the first eigenfrequency of the in-phase mode. Fig. 4 shows the dependence of the eigenfrequencies on the distance $L$ for different natural modes while Fig. 5 shows the $i$-th out-of-

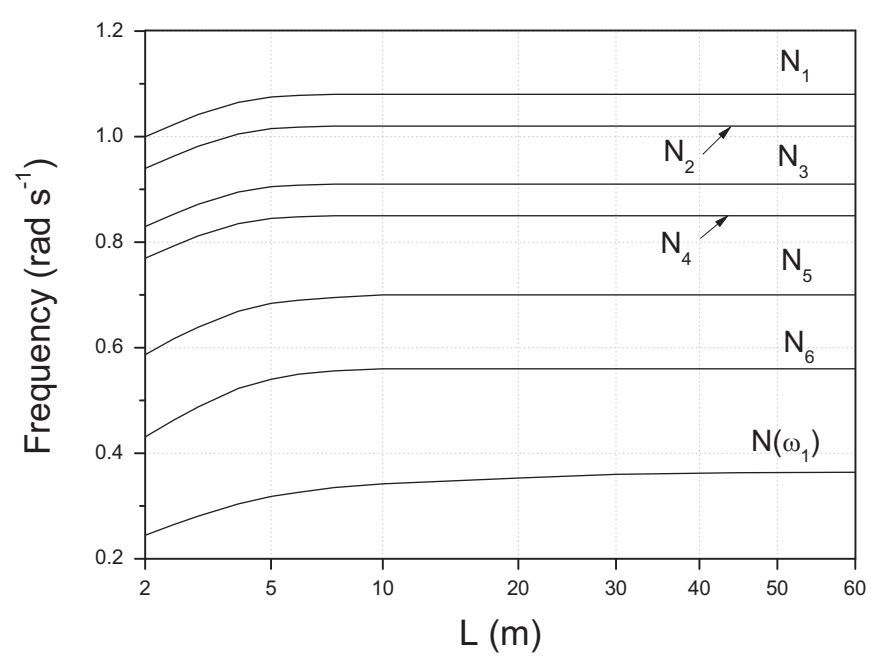

Fig. 4. Eigenfrequencies versus the distance $L$ for the different modal shapes. The eigenfrequencies tend to those of the single array in open sea for $L>10 \mathrm{~m}$.

phase modal profile $N_{i}$ described in Sammarco et al. (2013). In particular, $N_{1}, N_{3}$ and $N_{5}$ are symmetric with respect to the $x$ axis (even modes), $N_{2}, N_{4}$ and $N_{6}$ are antisymmetric (odd modes), while $N\left(\omega_{1}\right)$ is the first (even) in-phase natural mode. Only $N_{1}, N_{3}, N_{5}$ and $N\left(\omega_{1}\right)$ can be excited by incident waves orthogonal to the array. Similar results are obtained in Michele et al. (2015) for multiple array of gates in open sea.

Referring to Fig. 4, consider values of $L$ larger than $10 \mathrm{~m}$; the values of the eigenfrequencies remain substantially the same and 
a

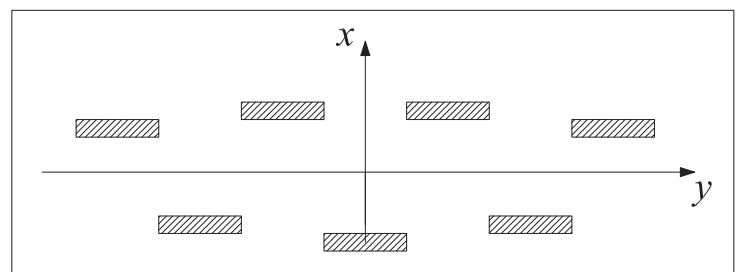

C
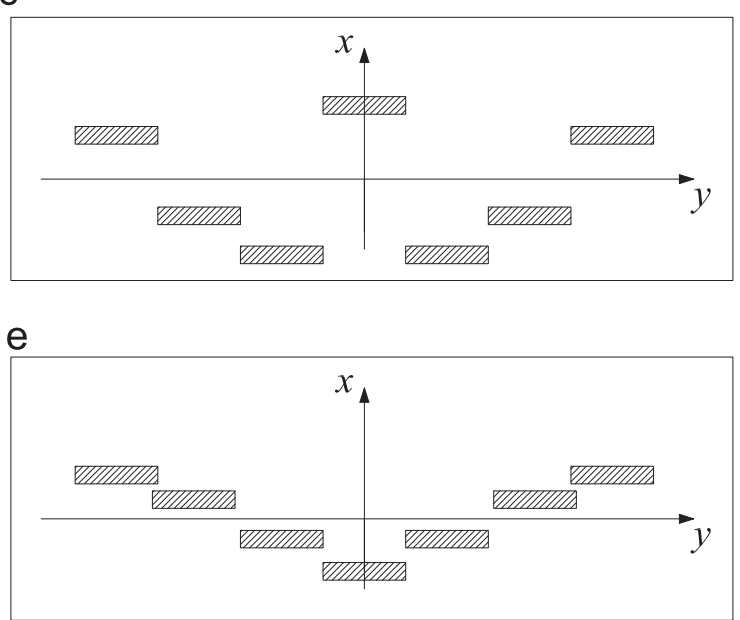

b

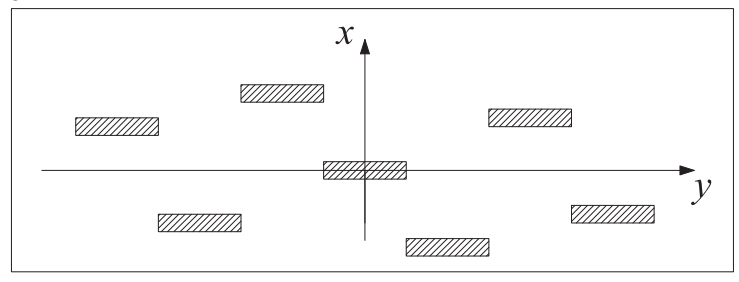

d
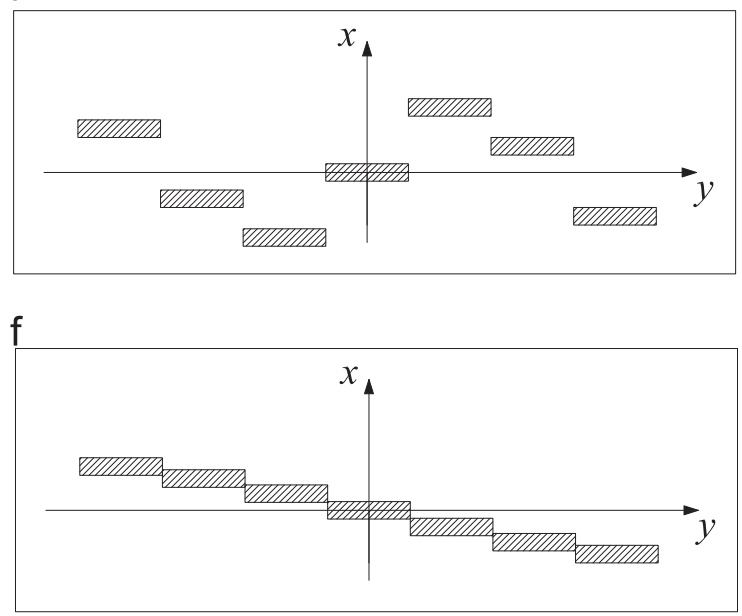

Fig. 5. Sketch of the out-of-phase modal profiles. (a) $N_{1}$, (b) $N_{2}$, (c) $N_{3}$, (d) $N_{4}$, (e) $N_{5}$ and (f) $N_{6}$.

tend to the eigenfrequencies of a single array in open sea. This is because for $L$ sufficiently large the gate motion evanescent terms do not interact with the vertical wall, and so the added inertia coincides with the open sea limit case $(L \rightarrow \infty)$.

\subsubsection{Exciting torque}

The variation of the absolute value of the exciting torque on each gate $G_{q}$ versus the incident wave frequency for $L=15 \mathrm{~m}$ and $L=30 \mathrm{~m}$ is shown in Fig. 6 . As in the case of one gate in front of the wall, local maxima and minima of $|F|$ which correspond respectively to the nodes and the antinodes of the standing wave field occur. Note that if the distance $L$ increases the number of the peaks in a fixed range of frequencies increases.

a

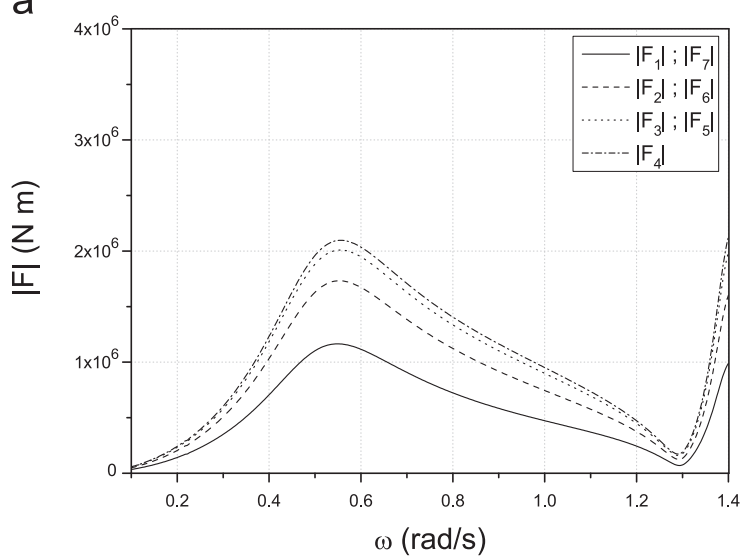

\subsubsection{Forced response}

Here we shall analyse the forced response of the array for a wide range of frequencies $\omega=0.1-1.4 \mathrm{rad} \mathrm{s}^{-1}$. The amplitude of the incident wave is assumed $A=1 \mathrm{~m}$. Fig. 7 shows the Response Amplitude Operator (RAO), i.e. the behaviour of amplitude of rotations $|\Theta|$ of each gate $G_{q}$ for varying frequency of the incident waves. As anticipated in previous Section, resonance occurs at frequencies that correspond to the even modes. Indeed, response peaks are located very close to the values of the eigenfrequencies; for these frequencies, radiation damping is small and the out-ofphase natural modes are nearly trapped. Note also that the sharpness and the unrealistic values of the peaks are symptom of weakness of the radiation damping. A similar behaviour of the amplitude of rotations is obtained in the case of the gate farm in open sea (Michele et al., 2015).

b

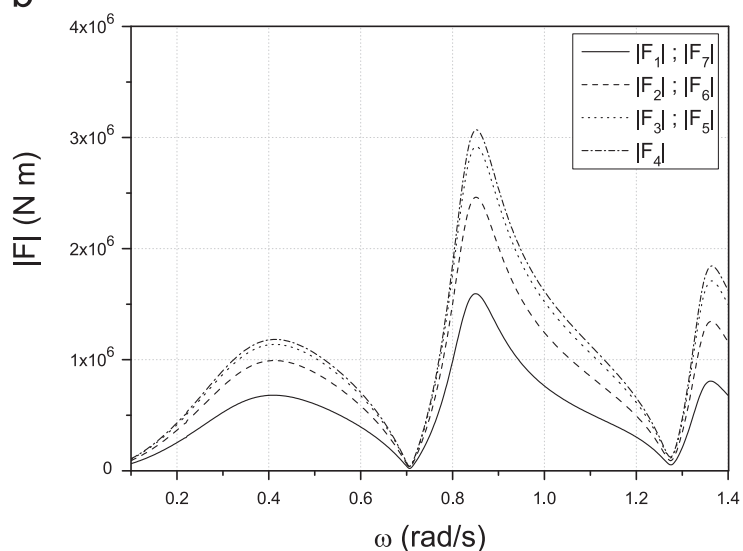

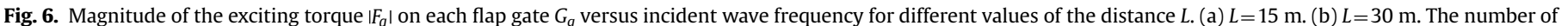
the maxima and minima increases with $L$. 
a

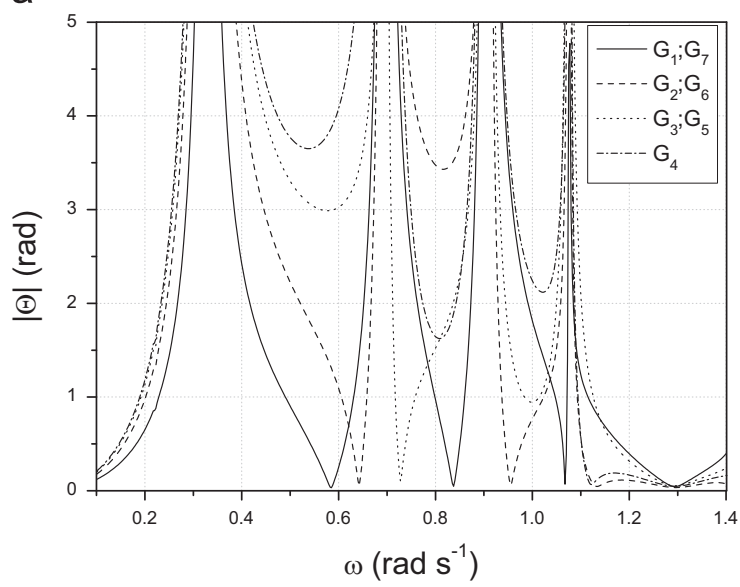

b



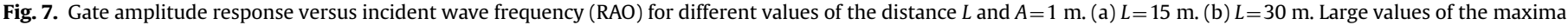
and sharpness of the peaks suggest that the radiation damping is small.

\subsection{Generated power and efficiency in monochromatic waves}

In order to evaluate the effects of the distance $L$ on the generated power $P$, a parametric analysis is performed for a wide range of frequencies. The efficiency of the system is assessed by considering the capture width ratio $C_{F}$, defined as the ratio of the generated power per unit array width to the incident power per unit width of the crest:

$$
C_{F}=\frac{P}{\frac{1}{2} \rho g A^{2} C_{g} w},
$$

where $C_{g}$ is the group velocity of the incident waves

$$
C_{g}=\frac{\omega}{2 k}\left(1+\frac{2 k h}{\operatorname{sh} 2 k h}\right) \text {, }
$$

$P$ is given by (53) and $w$ is the total width of the array. Fig. 8 shows the surface plots of the capture width ratio versus the incident wave frequency $\omega \in[0.1 ; 1.4] \mathrm{rad} \mathrm{s}^{-1}$ and the power-take off coefficients $\nu_{\text {pto }} \in\left[10^{2} ; 10^{8}\right] \mathrm{kg} \mathrm{m}^{2} \mathrm{~s}^{-1}$. For large values of $\nu_{p t o}$ maxima and minima are determined by the behaviour of the exciting torque shown in Fig. 6 and $C_{F}$, hence the performance of the array is dominated by the diffraction wave field. On the other hand, values $C_{F}<0.1$ are related to the minima of the exciting torque. Note that the maximum values of $C_{F}$ are more than seven times the values reached by the gate array in open sea.

a

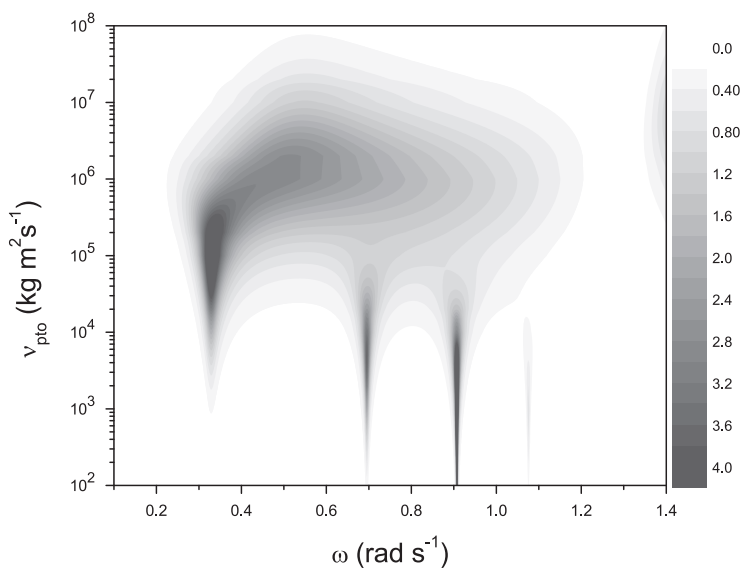

5.4.1. The array versus the single gate

We now compare in terms of wave energy production the array of $Q=7$ gates with the same gates locked in phase and one single isolated gate out of the seven. The distance between the array (gate) and the vertical wall is fixed to $L=15 \mathrm{~m}$. Hereafter, we optimize the power output of each system for $\omega=0.33 \mathrm{rad} \mathrm{s}^{-1}$, i.e. in correspondence of the eigenfrequency of the in-phase mode $N\left(\omega_{1}\right)$. Also the system of seven locked gates and the single gate must be tuned to resonance. Recall that these two systems are characterized by a single eigenfrequency. The numerical values of inertia and buoyancy which satisfy the resonance condition are listed in Table 3. Optimization of the power output yields $\nu_{\text {pto }}=10^{5} \mathrm{~kg} \mathrm{~m}^{2} \mathrm{~s}^{-1}$ for each gate of the array (see also Fig. 8(a)), $\nu_{\text {pto }}=7 \times 10^{5} \mathrm{~kg} \mathrm{~m}^{2} \mathrm{~s}^{-1}$ for seven locked gates and $\nu_{\text {pto }}=5 \times 10^{3} \mathrm{~kg} \mathrm{~m}^{2} \mathrm{~s}^{-1}$ for the single gate.

Fig. 9 shows the behaviour of $C_{F}$ versus the incident wave frequencies of each system when the power-take-off coefficients are optimized for $\omega \sim 0.33 \mathrm{rad} \mathrm{s}^{-1}$. Overall $C_{F}$ of the array is always larger than $C_{F}$ of the other systems. Consider the interval of $\omega \in[0.5 ; 1.2]$; this range includes the eigenfrequencies of the outof-phase modes of the array which amplify the response of the flaps and therefore the efficiency of the system. Note that all the eigenfrequencies (in-phase and out-of-phase) fall near the maximum of $|F|$ (see Fig. 6(a)). We have attained a summation of factors: the exciting torque is maximized, the array of gates is tuned to resonance and hence the generated power is maximized. Indeed

b

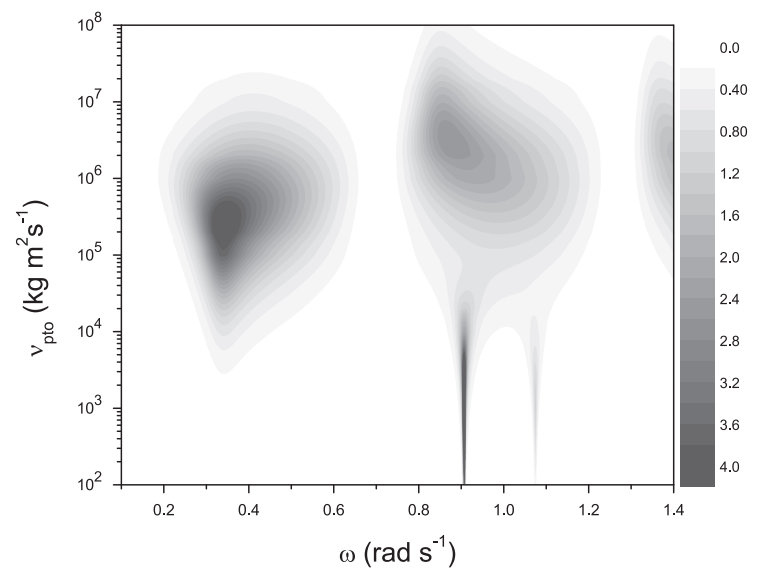

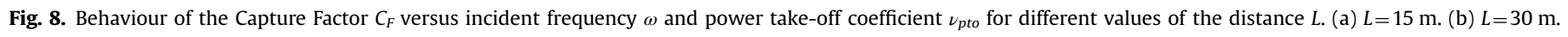


Table 3

Inertia and buoyancy of each system satisfying the resonance condition for $\omega=0.33 \mathrm{rad} \mathrm{s}^{-1}$

\begin{tabular}{lll}
\hline Gate system & Inertia $\left(\mathrm{kg} \mathrm{m}^{2}\right)$ & Buoyancy $\left(\mathrm{kg} \mathrm{m}^{2} \mathrm{~s}^{-1}\right)$ \\
\hline 7 locked gates & $7 \times 72 \times 10^{3}$ & $7 \times 3 \times 10^{5}$ \\
Single gate & $32 \times 10^{4}$ & $75 \times 10^{3}$ \\
\hline
\end{tabular}

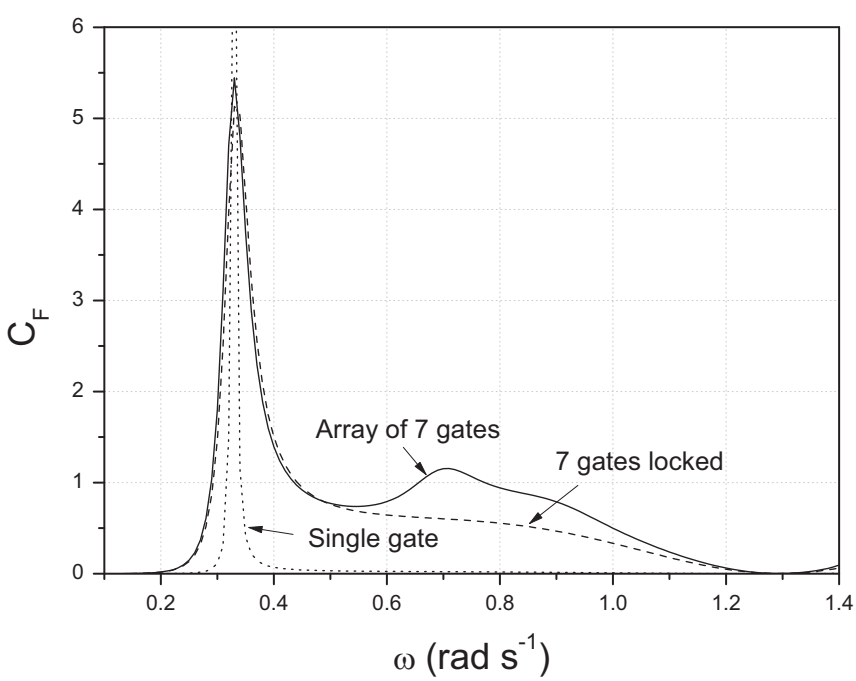

Fig. 9. Capture Factor $C_{F}$ of each system versus incident wave frequency $\omega$ for $L=15 \mathrm{~m}$. The PTO coefficients are optimized for $\omega=0.33 \mathrm{rad} \mathrm{s}^{-1}$.

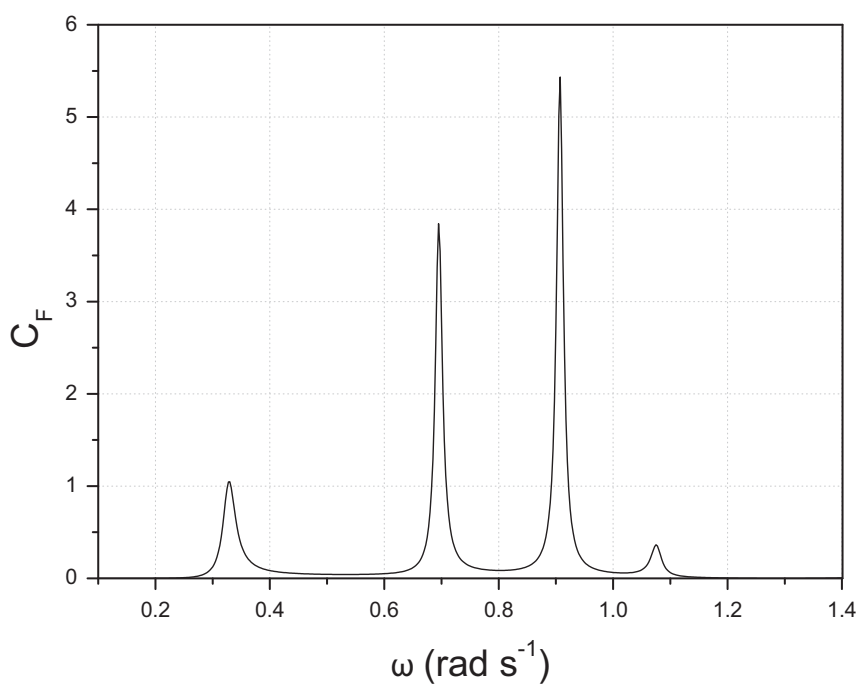

Fig. 10. Capture Factor $C_{F}$ of the array versus incident wave frequency $\omega$ for $L=15 \mathrm{~m}$. The PTO coefficient of each gate is optimized for the out-of-phase eigenfrequencies.

in optimal conditions the generated power depends on the ratio between the square of the exciting torque and the radiation damping $\nu$ which must be equal to $\nu_{\text {pto }}$ (Mei et al., 2005). As a consequence, large values of $|F|$ yield large values of $C_{F}$ and the system achieves the maximum efficiency. Another advantage is given by the fact that the array can be optimized also in correspondence of the out-of-phase modes. In this case, the optimal PTO coefficient is given by $\nu_{\text {pto }}=5 \times 10^{3} \mathrm{~kg} \mathrm{~m}^{2} \mathrm{~s}^{-1}$ and $C_{F}$ behaves as shown in Fig. 10. Note that these resonant peaks might not couple well with a random incident wave spectrum. In this case the efficiency of the array may be affected significantly. This aspect is investigated in the next section.

In summary, simply operating on $L$ and $\nu_{\text {pto }}$ the efficiency can



Fig. 11. Capture Factor $C_{F}$ of the array of $Q=3,5,7$ and 9 gates versus incident wave frequency $\omega$ for $L=15 \mathrm{~m}$. The PTO coefficients are optimized for $\omega=0.33 \mathrm{rad} \mathrm{s}^{-1}$.

be maximized for all the eigenfrequencies of the array. The behaviour of the exciting torque varies strongly with $L$ while for a fixed $I$ and $C$ the eigenfrequencies remain almost unchanged (see Fig. 4), hence, the tuning between $|F|$ and natural modes can be obtained by varying the distance of the array from the wall.

\subsubsection{Optimization of the number of the gates in an array}

In this section we compare the array of seven gates with the arrays of $Q=3,5,9$ gates having total width $w=21 \mathrm{~m}, L=15 \mathrm{~m}$ and eigenfrequency of the in-phase mode $\omega \sim 0.33 \mathrm{rad} \mathrm{s}^{-1}$. To maximize the power output of the array of $Q$ gates, inertia $I$, buoyancy $C$ and PTO coefficient $\nu_{\text {pto }}$ have been chosen respectively equal to $I=72000 \times 7 / Q \mathrm{~kg} \mathrm{~m}^{2}, C=300000 \times 7 / Q \mathrm{~kg} \mathrm{~m}^{2} \mathrm{~s}^{-2}$ and $\nu_{\text {pto }}=10^{5} \times 7 / \mathrm{Q} \mathrm{kg} \mathrm{m} \mathrm{s}^{-1}$. Fig. 11 shows the capture width ratio of each case versus incident wave frequency. The curves are very similar, hence if $Q \geq 3$ the power output does not change significantly.

\subsection{Generated power and efficiency in random seas}

In this Section we analyse the behaviour of the array in random seas. Assume that the wave field is represented by the one-dimensional JONSWAP spectrum (Hasselmann et al., 1973). The direction of the incident waves is orthogonal to the $y$-axis. The JONSWAP spectrum $S_{\zeta}(\omega)$ can be written in approximate form in terms of the significant wave height $H_{s}$ and peak frequency $\omega_{p}$ (Goda, 2012):

$S_{\zeta}(\omega)=\frac{\alpha H_{s}^{2}}{\omega}\left(\frac{\omega_{p}}{\omega}\right)^{4} \exp \left[-1,25\left(\frac{\omega_{p}}{\omega}\right)^{4}\right] \gamma^{\exp \left[-\left(\omega / \omega_{p}-1\right)^{2} /(2 \sigma)\right],}$

in which:

$\alpha=\frac{0.0624}{0.23+0.0336 \gamma-0.185(1,9+\gamma)^{-1}}(1,094-0.01915 \ln \gamma)$,

$\sigma=\left\{\begin{array}{l}0.07: \omega \leq \omega_{p} \\ 0.09: \omega>\omega_{p}\end{array}\right.$,

$\gamma=3.3$

The angular displacement $\Theta_{q}(t)$ of the gate $G_{q}$ can be related to the incident wave field spectrum through the relation (Babarit et al., 2012): 
$\Theta_{q}(t)=\sum_{n=1}^{\infty}\left|\theta_{q}\right| \sqrt{2 S_{\zeta}\left(\omega_{n}\right) d \omega} \cos \left(\omega_{n} t+\delta_{n}\right), \quad q=1, \ldots, Q$,

in which $d \omega$ is an adequate frequency step, $\delta_{n}$ are a set of random phases and $\left|\theta_{q}\right|$ represents the amplitude response of the gate $G_{q}$ for $A=1 \mathrm{~m}$ in the frequency domain. Once the $q$-th gate angular displacement is known, the instantaneous generated power $P_{q}$ can be written as:

$P_{q}(t)=\nu_{p t o}\left(\sum_{n=1}^{\infty}\left|\theta_{q}\right| \sqrt{2 S_{\zeta}\left(\omega_{n}\right) d \omega} \sin \left(\omega_{n} t+\delta_{n}\right) \omega_{n}\right)^{2}$,

while the average extracted power over a period $T_{R}$ (Eriksson et al., 2005) of the entire array is given by:

$\bar{P}=\frac{1}{T_{R}} \int_{0}^{T_{R}} \sum_{q=1}^{Q} P_{q} d t$.

If $T_{R}$ is large, expression (64) yields (see also Sarkar et al., 2013):

$\bar{P} \simeq \nu_{\text {pto }} \sum_{q=1}^{Q} \sum_{n=1}^{\infty}\left|\theta_{q}\right|^{2} \omega_{n}^{2} S_{\zeta}\left(\omega_{n}\right) d \omega$.

The capture width ratio in real seas $C_{F \zeta}$ can be defined as the ratio of the mean power extracted from the array to the incident wave power related to the spectrum $S_{\zeta}$ :

$C_{F \zeta}=\frac{\bar{P}}{w P_{\zeta}}$,

where:

$P_{\zeta}=\sum_{n=1}^{\infty} C_{g}\left(\omega_{n}\right) \rho g S_{\zeta}\left(\omega_{n}\right) d \omega$

is the incident power per unit crest width, while $C_{g}\left(\omega_{n}\right)$ is the group velocity of the generic component $\omega_{n}$. Expression (66) gives the capture width ratio for any sea state characterized by $H_{s}$ and peak frequency $\omega_{p}$. Fig. 12 shows $C_{F \zeta}$ versus peak frequency for three values of the PTO-coefficient. By choosing $\nu_{\text {pto }}=10^{5} \mathrm{~kg} \mathrm{~m}^{2} \mathrm{~s}^{-1}$ we optimize the power output for the in-phase eigenfrequency $\omega_{p}=0.33 \mathrm{rad} \mathrm{s}^{-1}$ (continuous line). $C_{F \zeta}$ has a smaller peak than that of the capture width ratio $C_{F}$ shown in Fig. 9 and reduces from 5 to 3. By increasing the PTO-coefficient to $\nu_{\text {pto }}=10^{6} \mathrm{~kg} \mathrm{~m}^{2} \mathrm{~s}^{-1}$ (dashed line) the diffraction wave field dominates the dynamics of the array and the behaviour of $C_{F \zeta}$ resembles that of the magnitude of

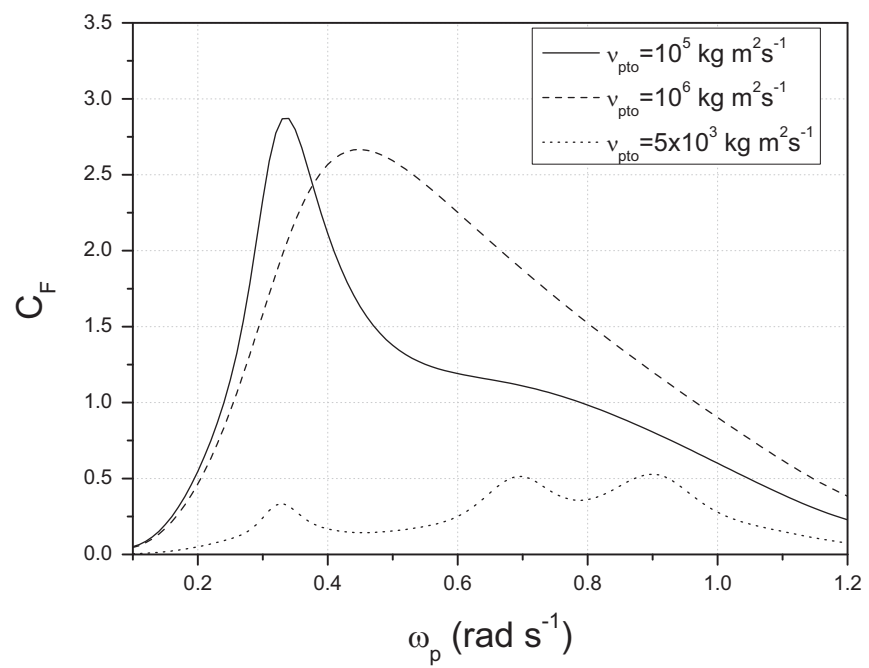

Fig. 12. Capture Factor in real seas $C_{F \zeta}$ versus peak frequency $\omega_{p}$ for different values of the power take-off coefficient. the exciting torque shown in Fig. 6(a). Finally, decreasing the PTOcoefficient to $\nu_{\text {pto }}=5 \times 10^{3} \mathrm{~kg} \mathrm{~m}^{2} \mathrm{~s}^{-1}$ (dotted line) the out-of-phase resonance peaks become smaller (yet still meaningful) than those shown in Fig. 10 for monochromatic waves. This is because the incident wave spectrum does not couple well with the narrow peaks. In this case it is more convenient to choose larger values of $\nu_{\text {pto }}$ which give a better performance across a larger frequency interval.

\section{Conclusions}

We analysed an array of gates of finite thickness in front of a vertical wall. The method of solution adopted is similar to that of Michele et al. (2015). Resorting to Green's theorem and Green's function, the radiation and scattering problems are solved via a semi-analytical approach. To take into account the boundary condition on the vertical wall, the method of images has been applied. The radiation and scattering potentials on the boundary of the array are expressed in terms of Legendre polynomials and the respective coefficients have been obtained by the solution of a system of hypersingular integral equations.

Unlike a single or a multiple series of array in open sea, the array in front of a vertical wall can exploit both the resonance of the natural modes and the enhancement of the exciting torque. The tuning between eigenfrequencies and maximum peaks of the exciting torque can be viewed as the optimal condition to extract energy from incident waves. Indeed in this case, the maximum value of the capture width ratio is much larger than the value reached by the array in open sea, hence the presence of the wall can be viewed as a useful method for capturing more power. This is indeed our current ongoing research to find a new optimal device. Finally the behaviour of the array in presence of incident waves represented by the JONSWAP spectrum is investigated. When in irregular waves the maxima of the capture width ratio are smaller than the maxima of the capture width ratio in monochromatic waves.

This analysis is performed in the framework of ideal fluid and irrotational flow; viscous effects and vortex shedding may modify the response of the array and will be considered in future works.

\section{Appendix A. Solution of the radiation and scattering potentials}

Define $x_{A}$ and $\xi_{A}$ as follows:

$x_{A}=x-L, \quad \xi_{A}=\xi-L$,

and introduce the dimensionless variables denoted by primes:

$\eta^{\prime}=\frac{2 \eta}{w}, \quad y^{\prime}=\frac{2 y}{w}, \quad \xi^{\prime}=\frac{\xi_{A}}{b}, \quad x^{\prime}=\frac{x_{A}}{b}$.

The radiation and the scattering potentials on the boundary of the array, can be expressed in terms of the new function $f$ and $g$ each defined in the interval $[-1,1]$ :

$\left\{\begin{array}{l}\varphi_{n, q}^{R}\left(\xi=\xi^{ \pm}, \eta\right) \\ \varphi_{n}^{S}\left(\xi=\xi^{ \pm}, \eta\right)\end{array}\right\}=\left\{\begin{array}{l}\varphi_{n, p q}^{R}\left(\xi_{A}= \pm b, \eta\right) \\ \varphi_{n}^{S}\left(\xi_{A}= \pm b, \eta\right)\end{array}\right\}=\left\{\begin{array}{l}f_{n, q}^{R \pm}\left(\eta^{\prime}\right) \\ f_{n}^{S_{ \pm}}\left(\eta^{\prime}\right)\end{array}\right\}$,

$\left\{\begin{array}{c}\varphi_{n, q}^{R}\left(\xi, \eta= \pm \frac{w}{2}\right) \\ \varphi_{n}^{S}\left(\xi, \eta= \pm \frac{w}{2}\right)\end{array}\right\}=\left\{\begin{array}{l}g_{n, q}^{R_{ \pm}}\left(\xi^{\prime}\right) \\ g_{n}^{S_{ \pm}}(\xi)\end{array}\right\}$. 
Apply the change of variables (A.1) and (A.2) to the expressions (38) and (39) and define the following integrals for shorthand notation:

$\left\{\begin{array}{c}\mathcal{W}_{n, q}^{R \pm} \\ \mathcal{W}_{n}^{S_{ \pm}}\end{array}\right\}=\left.\mp \frac{W}{2 b} \int_{-1}^{1}\left\{\begin{array}{l}f_{n, q}^{R_{ \pm}} \\ f_{n}^{S_{ \pm}}\end{array}\right\} \frac{\partial G_{n}}{\partial \xi^{\prime}}\right|_{\xi^{\prime}= \pm 1} d \eta^{\prime}$,

$\left\{\begin{array}{l}\mathcal{B}_{n, q}^{R_{ \pm}} \\ \mathcal{B}_{q^{S}}^{S_{ \pm}}\end{array}\right\}=\left.\mp \frac{2 b}{w} \int_{-1}^{1}\left\{\begin{array}{l}g_{n, q}^{R_{ \pm}} \\ g_{n}^{S_{ \pm}}\end{array}\right\} \frac{\partial G_{n}}{\partial \eta^{\prime}}\right|_{\eta^{\prime}= \pm 1} d \xi^{\prime}$

$\mathcal{W}_{n, q}^{R}=\mathrm{i} \omega \theta_{q} f_{n} \frac{w}{2} \int_{\frac{2 \eta q}{w}}^{\frac{2 \eta_{q+1}}{w}}\left[\left.G_{n}\right|_{\xi^{\prime}=-1}-\left.G_{n}\right|_{\xi^{\prime}=1}\right] d \eta^{\prime}$,

$\mathcal{W}_{n}^{S}=A d_{n} \frac{w}{2} \int_{-1}^{1}\left[\left.\sin \left[k_{n}\left(\xi^{\prime} b+L\right)\right] G_{n}\right|_{\xi^{\prime}=1}-\left.\sin \left[k_{n}\left(\xi^{\prime} b+L\right)\right] G_{n}\right|_{\xi^{\prime}=-1}\right] d \eta^{\prime}$,

Imposing the boundary conditions (25)-(28) to the radiation and scattering potentials (38) and (39) yields:

$\frac{\partial}{\partial x^{\prime}}\left[\mathcal{W}_{n, q}^{R+}+\mathcal{W}_{n, q}^{R-}+\mathcal{B}_{n, q}^{R+}+\mathcal{B}_{n, q}^{R-}+\mathcal{W}_{n, q}^{R}\right]$

$=\left\{\begin{array}{lll}-\frac{\mathrm{i} \omega \theta_{q} f_{n} b}{2} & \text { on } S_{q}^{\prime}, & \text { (a) } \\ 0 & \text { on } S_{\tilde{q}}^{\prime}, \quad \tilde{q} \neq q, & \text { (b) }\end{array}\right.$

$\frac{\partial}{\partial y^{\prime}}\left[\mathcal{W}_{n, q}^{R+}+\mathcal{W}_{n, q}^{R-}+\mathcal{B}_{n, q}^{R+}+\mathcal{B}_{n, q}^{R-}+\mathcal{W}_{n, q}^{R}\right]=0 \quad$ on $S_{w}^{\prime}$

(A.9c)

$\frac{\partial}{\partial x^{\prime}}\left[\mathcal{W}_{n}^{+S}+\mathcal{W}_{n}^{S-}+\mathcal{B}_{n}^{+S}+\mathcal{B}_{n}^{+S}+\mathcal{W}_{n}^{S}\right]$

$=\frac{A d_{n} \sin \left(k_{n} X^{ \pm}\right) b}{2}$ on $S_{\tilde{q}}^{\prime}$,

(A.10a)

$\frac{\partial}{\partial y^{\prime}}\left[\mathcal{W}_{n}^{+S}+\mathcal{W}_{n}^{S-}+\mathcal{B}_{n}^{+S}+\mathcal{B}_{n}^{+S}+\mathcal{W}_{n}^{S}\right]=0$ on $S_{w}^{\prime}$,

(A.10b)

$\tilde{q}=1, \ldots, Q$.

where $S_{q}^{\prime}$ and $S_{w}^{\prime}$ represent the normalized boundaries:

$S_{q}^{\prime}=\left\{x^{\prime}= \pm 1, y \in\left(\frac{2 y_{q}}{w}, \frac{2 y_{q+1}}{w}\right)\right\}$,

$S_{w}^{\prime}=\left\{x^{\prime} \in(-1,1), y^{\prime}= \pm 1\right\}$,

Expressions (A.9a)-(A.10b) form two systems of 4 integro-differential equations whose unknowns are respectively $\varphi_{n, q}^{R}$ and $\varphi_{n}{ }^{S}$ evaluated on the boundary of the array. However, the integrals inside (A.9a)-(A.10b), given by

$\frac{\partial}{\partial x}\left\{\begin{array}{c}\mathcal{W}_{n, q}^{R_{ \pm}} \\ \mathcal{W}_{n}^{S_{ \pm}}\end{array}\right\}, \quad \frac{\partial}{\partial y}\left\{\begin{array}{l}\mathcal{B}_{n, q}^{R_{ \pm}} \\ \mathcal{B}_{n}^{S_{ \pm}}\end{array}\right\}$

are hypersingular when $\eta^{\prime}= \pm y^{\prime}$ and $\xi^{\prime}= \pm x^{\prime}$. In this case, the inversion between the outer derivative and the integral sign is possible if the latter is interpreted as a Hadamard finite-part integral $\mathrm{H} \int$.
Recalling the expression of the Hankel function $H_{1}^{(1)}$ (Gradshteyn and Ryzhik, 2007, 8.444)

$H_{1}^{(1)}(\alpha)=-\frac{2 \mathrm{i}}{\alpha \pi}+R_{n}(\alpha)$

where:

$R_{n}(\alpha)=J_{1}(\alpha)+\frac{\mathrm{i}}{\pi}\left\{2 J_{1}(\alpha)\left(\frac{\ln \alpha}{2}+\gamma\right)-\frac{\alpha}{2}-\sum_{k=2}^{\infty}(-1)^{k+1} \frac{(\alpha / 2)^{2 k-1}}{k !(k-1) !}\left(\frac{1}{k}+2 \sum_{m=1}^{k-1} \frac{1}{m}\right)\right\}$,

with $J_{1}(\alpha)$ the Bessel function of the first kind and order 1 and $\gamma$ the Euler-Mascheroni constant. When the integrals in (A.13) becomes hypersingular can be rewritten as:

$\frac{\partial}{\partial x}\left\{\begin{array}{c}\mathcal{I}_{n, q}^{R_{ \pm}} \\ \mathcal{I}_{n}^{S_{ \pm}}\end{array}\right\}= \pm \frac{1}{w \pi} \mathrm{H} \int_{-1}^{1}\left\{\begin{array}{l}f_{n, q}^{R_{ \pm}} \\ f_{n}^{S_{ \pm}}\end{array}\right\}\left(y^{\prime}-\eta^{\prime}\right)^{-2} d \eta^{\prime} \mp\left\{\begin{array}{c}\mathcal{L}^{R_{ \pm}}\left(f_{n, q}^{R_{ \pm}}\right) \\ \mathcal{L}^{S_{ \pm}}\left(f_{n}^{S_{ \pm}}\right)\end{array}\right\}$

on $S_{\tilde{q}}^{\prime}$,

$\frac{\partial}{\partial y}\left\{\begin{array}{l}\mathcal{H}_{n, q}^{R \pm} \\ \mathcal{H}_{n}^{S_{ \pm}}\end{array}\right\} \pm \frac{1}{2 \pi b} \mathrm{H} \int_{-1}^{1}\left\{\begin{array}{l}g_{n, q}^{R_{ \pm}} \\ g_{n}^{S_{ \pm}}\end{array}\right\}\left(x^{\prime}-\xi^{\prime}\right)^{-2} d \xi^{\prime} \mp\left\{\begin{array}{l}\mathcal{T}^{R_{ \pm}}\left(g_{n, q}^{R_{ \pm}}\right) \\ \mathcal{T}^{S_{ \pm}}\left(g_{n}^{S_{ \pm}}\right)\end{array}\right\}$

on $S_{w}^{\prime}$,

where a change of notations is executed to distinguish non-singular integrals ( $\mathcal{W}$ an $\mathcal{B}$ ) and hypersingular integrals $(\mathcal{I}$ and $\mathcal{H}$ ). The non-singular integrals $\mathcal{L}$ an $\mathcal{T}$ inside (A.16) and (A.17) can be written as:

$$
\begin{aligned}
\left\{\begin{array}{c}
\mathcal{L}^{R \pm}\left(f_{n, q}^{R \pm}\right) \\
\mathcal{L}^{S_{ \pm}}\left(f_{n}^{S_{ \pm}}\right)
\end{array}\right\}= & \int_{-1}^{1}\left\{\begin{array}{c}
f_{n, q}^{R \pm} \\
f_{n}^{S_{ \pm}}
\end{array}\right\}_{\xi^{\prime}= \pm 1} \frac{k_{n} \mathrm{i} R_{n}\left(k_{n}\left|y^{\prime}-\eta^{\prime}\right| \frac{w}{2}\right)}{4\left|y^{\prime}-\eta^{\prime}\right|} d \eta^{\prime} \\
& +\left.\frac{w}{2 b} \int_{-1}^{1}\left\{\begin{array}{c}
f_{n, q}^{R \pm} \\
f_{n}^{S_{ \pm}}
\end{array}\right\} \frac{\partial G_{n}^{(1)}}{\partial \xi^{\prime}}\right|_{\xi^{\prime}= \pm 1} d \eta^{\prime},
\end{aligned}
$$

$$
\begin{aligned}
\left\{\begin{array}{c}
\mathcal{T}^{R_{ \pm}}\left(g_{n, q}^{R_{ \pm}}\right) \\
\mathcal{T}^{S_{ \pm}}\left(g_{n}^{S_{ \pm}}\right)
\end{array}\right\}= & \int_{-1}^{1}\left\{\begin{array}{l}
g_{n, q}^{R_{ \pm}} \\
g_{n}^{S_{ \pm}}
\end{array}\right\}_{\eta^{\prime}= \pm 1} \frac{k_{n} \mathrm{i} R_{n}\left(k_{n}\left|x^{\prime}-\xi^{\prime}\right| b\right)}{4\left|x^{\prime}-\xi^{\prime}\right|} d \xi^{\prime} \\
& +\left.\frac{2 b}{w} \int_{-1}^{1}\left\{\begin{array}{l}
g_{n, q}^{R_{ \pm}} \\
g_{n}^{S_{ \pm}}
\end{array}\right\} \frac{\partial G_{n}^{(1)}}{\partial \eta^{\prime}}\right|_{\eta^{\prime}= \pm 1} d \xi^{\prime} .
\end{aligned}
$$

Note that both $\mathcal{L}$ and $\mathcal{T}$ have a convergent kernel. Indeed, when $\left|y^{\prime}-\eta^{\prime}\right| \rightarrow 0$ and $\left|x^{\prime}-\xi^{\prime}\right| \rightarrow 0, \quad R_{n}\left(k_{n}\left|y^{\prime}-\eta^{\prime}\right| \frac{w}{2}\right) \simeq\left|y^{\prime}-\eta^{\prime}\right| \ln \left|y^{\prime}-\eta^{\prime}\right|$ and $R_{n}\left(k_{n}\left|x^{\prime}-\xi^{\prime}\right| b\right) \simeq\left|x^{\prime}-\xi^{\prime}\right| \ln \left|x^{\prime}-\xi^{\prime}\right|$, hence the syngularity is isolated only in the finite part-integral.

The structure of the hypersingular integrals suggests to seek solutions of the type:

$\left\{\begin{array}{c}f_{n, q}^{R \pm} \\ f_{n}^{S \pm}\end{array}\right\}=\sum_{m=0}^{M}\left\{\begin{array}{c}\alpha_{n m}^{R \pm q}, P_{m} \theta_{q} \\ \alpha_{n m}^{S_{ \pm}} P_{m}\end{array}\right\}$

(A.20)

$\left\{\begin{array}{c}g_{n, q}^{R_{ \pm}} \\ g_{n}^{S_{ \pm}}\end{array}\right\}=\sum_{m=0}^{M}\left\{\begin{array}{c}\beta_{n m, q}^{R_{ \pm}} P_{m} \theta_{q} \\ \beta_{n m}^{S_{ \pm}} P_{m}\end{array}\right\}$,

Substituting the series expansions (A.20) and (A.21) into the hypersingular integrals of (A.16) and (A.17), yields: 


$$
\begin{aligned}
& \mathrm{H} \int_{-1}^{1}\left\{\begin{array}{c}
f_{n, q}^{R \pm} \\
f_{n}^{S_{ \pm}}
\end{array}\right\}\left(y^{\prime}-\eta^{\prime}\right)^{-2} d \eta^{\prime} \\
& =\sum_{m=0}^{M}\left\{\begin{array}{c}
\alpha_{n m, q}^{R_{ \pm}} \theta_{q} \\
\alpha_{n m}^{S_{ \pm}}
\end{array}\right\}\left[-2(m+1) \frac{y^{\prime} Q_{m}\left(y^{\prime}\right)-Q_{m+1}\left(y^{\prime}\right)}{1-y^{\prime 2}}\right] \\
& \mathrm{H} \int_{-1}^{1}\left\{\begin{array}{c}
g_{n, q}^{R_{ \pm}} \\
g_{n}^{S_{ \pm}}
\end{array}\right\}\left(x^{\prime}-\xi^{\prime}\right)^{-2} d \xi^{\prime} \\
& =\sum_{m=0}^{M}\left\{\begin{array}{c}
\beta_{n m, q}^{R_{ \pm}} \theta_{q} \\
\beta_{n m}^{S_{ \pm}}
\end{array}\right\}\left[-2(m+1) \frac{x^{\prime} Q_{m}\left(x^{\prime}\right)-Q_{m+1}\left(x^{\prime}\right)}{1-x^{\prime 2}}\right] .
\end{aligned}
$$

Finally the hypersingular integrals are solved in terms of Legendre polynomials, hence (A.16) and (A.17) become:

$\left\{\begin{array}{c}\mathcal{I}_{n, q}^{R_{ \pm}} \\ \mathcal{I}_{n}^{S_{ \pm}}\end{array}\right\}=\sum_{m=0}^{M}\left\{\begin{array}{c}\alpha_{n m, q}^{R \pm} \theta_{q} \\ \alpha_{n \pm}^{S_{ \pm}}\end{array}\right\}\left\{\begin{array}{c}\widetilde{\mathcal{I}}_{m}^{R_{ \pm}} \\ \widetilde{\mathcal{I}}_{m}^{S_{ \pm}}\end{array}\right\}$,

$\left\{\begin{array}{c}\mathcal{H}_{n, q}^{R_{ \pm}} \\ \mathcal{H}_{n}^{S_{ \pm}}\end{array}\right\}=\sum_{m=0}^{M}\left\{\begin{array}{c}\beta_{n m, q}^{R_{ \pm}} \theta_{q} \\ \beta_{n m}^{S_{ \pm}}\end{array}\right\}\left\{\begin{array}{c}\widetilde{\mathcal{H}}_{m}^{R_{ \pm}} \\ \widetilde{\mathcal{H}}_{m}^{S_{ \pm}}\end{array}\right\}$,

where:

$$
\begin{gathered}
\left\{\begin{array}{c}
\widetilde{\mathcal{I}}_{m}^{R_{ \pm}} \\
\widetilde{\mathcal{I}}_{m}^{S_{ \pm}}
\end{array}\right\}=\mp \frac{2}{w \pi}\left[(m+1) \frac{y^{\prime} Q_{m}\left(y^{\prime}\right)-Q_{m+1}\left(y^{\prime}\right)}{1-y^{\prime 2}}\right] \mp\left\{\begin{array}{l}
\mathcal{L}^{R_{ \pm}}\left(P_{m}\right) \\
\mathcal{L}^{S_{ \pm}}\left(P_{m}\right)
\end{array}\right\}, \\
\left\{\begin{array}{l}
\widetilde{\mathcal{H}}_{m}^{R_{ \pm}} \\
\widetilde{\mathcal{H}}_{m}^{S_{ \pm}}
\end{array}\right\}=\mp \frac{1}{b \pi}\left[(m+1) \frac{x^{\prime} Q_{m}\left(x^{\prime}\right)-Q_{m+1}\left(x^{\prime}\right)}{1-x^{\prime 2}}\right] \mp\left\{\begin{array}{l}
\mathcal{T}^{R_{ \pm}}\left(P_{m}\right) \\
\mathcal{T}^{S_{ \pm}}\left(P_{m}\right)
\end{array}\right\} .
\end{gathered}
$$

The expressions (A.5) and (A.6) which include the functions $f$ and $g$, after substitution of (A.20) and (A.21) become:

$$
\begin{aligned}
\left\{\begin{array}{c}
\mathcal{W}_{n, q}^{R_{ \pm}} \\
\mathcal{W}_{n^{ \pm}}^{S_{ \pm}}
\end{array}\right\} & =\left.\mp \frac{w}{2 b} \sum_{m=0}^{M}\left\{\begin{array}{c}
\alpha_{n m, q}^{R_{ \pm}} \theta_{q} \\
\alpha_{n m}^{S_{ \pm}}
\end{array}\right\} \int_{-1}^{1} P_{m}\left(\eta^{\prime}\right) \frac{\partial G_{n}}{\partial \xi^{\prime}}\right|_{\xi^{\prime}= \pm 1} d \eta^{\prime} \\
& =\mp \frac{w}{2 b} \sum_{m=0}^{M}\left\{\begin{array}{c}
\alpha_{n m, q}^{R_{ \pm}} \theta_{q} \\
\alpha_{n m}^{S_{ \pm}}
\end{array}\right\}\left\{\begin{array}{c}
\widetilde{\mathcal{W}}_{m}^{R_{ \pm}} \\
\widetilde{\mathcal{W}}_{m}^{S_{ \pm}}
\end{array}\right\}, \\
\left\{\begin{array}{c}
\mathcal{B}_{n, q}^{R_{ \pm}} \\
\mathcal{B}_{n}^{S_{ \pm}}
\end{array}\right\} & =\left.\mp \frac{2 b}{w} \sum_{m=0}^{M}\left\{\begin{array}{c}
\beta_{n m, q}^{R_{ \pm}} \theta_{q} \\
\beta_{n m}^{S_{ \pm}}
\end{array}\right\} \int_{-1}^{1} P_{m}\left(\xi^{\prime}\right) \frac{\partial G_{n}}{\partial \eta^{\prime}}\right|_{\eta^{\prime}= \pm 1} d \xi^{\prime} \\
& =\mp \frac{2 b}{w} \sum_{m=0}^{M}\left\{\begin{array}{c}
\beta_{n m, q}^{R_{ \pm}} \theta_{q} \\
\beta_{n m}^{S_{ \pm}}
\end{array}\right\}\left\{\begin{array}{l}
\widetilde{\mathcal{B}}_{m}^{R_{ \pm}} \\
\widetilde{\mathcal{B}}_{m}^{S_{ \pm}}
\end{array}\right\} .
\end{aligned}
$$

Finally, the two system (A.9a)-(A.9c) and (A.10a)-(A.10b) can be rewritten as:

$$
\begin{array}{r}
\frac{\partial}{\partial x^{\prime}} \sum_{m=0}^{M} \alpha_{n m, q}^{R \mp} \theta_{q} \widetilde{\mathcal{W}}_{m}^{R \mp}+\beta_{n m, q}^{R+} \theta_{q} \widetilde{\mathcal{B}}_{m}^{R+}+\beta_{n m, q}^{R-} \theta_{q} \widetilde{\mathcal{B}}_{m}^{R-}+\mathcal{W}_{n, q}^{R} \\
+\alpha_{n m, q}^{R_{ \pm}} \theta_{q} \widetilde{\mathcal{I}}_{m}^{R_{ \pm}}= \begin{cases}-\frac{\mathrm{i} \omega \theta_{q} f_{n} b}{2} & \text { on } S_{q}^{\prime}, \quad \text { (a) } \\
0 & \text { on } S_{\tilde{q}}^{\prime}, \quad \tilde{q} \neq q,\end{cases}
\end{array}
$$

$$
\begin{aligned}
& \frac{\partial}{\partial y^{\prime}} \sum_{m=0}^{M} \beta_{n m, q}^{R \mp} \theta_{q} \widetilde{\mathcal{B}}_{m}^{R \mp}+\alpha_{n m, q}^{R+} \theta_{q} \widetilde{\mathcal{W}}_{m}^{R+}+\alpha_{n m, q}^{R-} \theta_{q} \widetilde{\mathcal{W}}_{m}^{R-}+\mathcal{W}_{n, q}^{R} \\
& \quad+\beta_{n m, q}^{R+} \theta_{q} \widetilde{\mathcal{H}}_{m}^{R \pm}=0, \quad \text { on } S_{w}^{\prime},
\end{aligned}
$$

$$
\begin{aligned}
& \frac{\partial}{\partial x^{\prime}} \sum_{m=0}^{M} \alpha_{n m}^{S_{\mp}} \widetilde{\mathcal{W}}_{m}^{S_{\mp}}+\beta_{n m}^{S+} \widetilde{\mathcal{B}}_{m}^{S+}+\beta_{n m}^{S-} \widetilde{\mathcal{B}}_{m}^{S-}+\mathcal{W}_{n}^{S}+\alpha_{n m}^{S_{ \pm}} \widetilde{\mathcal{I}}_{m}^{S_{ \pm}} \\
& \quad=\frac{A d_{n} \sin \left(k_{n} x^{ \pm}\right) b}{2}, \quad \text { on } S_{\tilde{q}}^{\prime},
\end{aligned}
$$$$
\frac{\partial}{\partial y^{\prime}} \sum_{m=0}^{M} \beta_{n m}^{S_{\mp}} \widetilde{\mathcal{B}}_{m}^{S_{\mp}}+\alpha_{n m}^{S+} \widetilde{\mathcal{W}}_{m}^{S+}+\alpha_{n m}^{S-} \widetilde{\mathcal{W}}_{m}^{S-}+\mathcal{W}_{n}^{S}+\beta_{n m}^{S_{ \pm}} \widetilde{\mathcal{H}}_{m}^{S_{ \pm}}
$$$$
=0 \text {, on } S_{w}^{\prime} \text {, }
$$

$\tilde{q}=1, \ldots, Q$.

Expressions (A.30a)-(A.30c) and (A.31a)-(A.31b) produce two systems of linear equations whose unknowns are respectively $\alpha_{n m, q}^{R_{ \pm}}$and $\beta_{n m, q}^{R_{ \pm}}$for the radiation problem, $\alpha_{n m}^{S_{ \pm}}$and $\beta_{n m}^{S_{ \pm}}$for the scattering problem. The number of unknowns is equal to $4 \times M+1$, hence $M+1$ evaluation points must be chosen for each side of the array. A good choice for the collocation points $\left(x_{j}^{\prime}, y_{j}^{\prime}\right)$ is given by the roots of Chebyshev polynomials of the first kind (Kaya and Erdogan, 1987; Parsons and Martin, 1992) i.e.

$\left(x_{j}^{\prime}, y_{j}^{\prime}\right)=\left(\cos \frac{(2 j+1) \pi}{2 M+2}, \pm 1\right), \quad$ on $S_{\tilde{q}}^{\prime}$

$\left(x_{j}^{\prime}, y_{j}^{\prime}\right)=\left( \pm 1, \cos \frac{(2 j+1) \pi}{2 M+2}\right), \quad$ on $S_{w}^{\prime}$

$\tilde{q}=1, \ldots, Q, \quad j=0,1, \ldots, M$

Systems (A.30a)-(A.30c) and (A.31a)-(A.31b) can be solved numerically for each modal order $n=0,1, \ldots$, therefore the radiation potential $\phi_{q}{ }^{R}$ and the scattering potential $\phi^{S}$ on the boundary of the array, are given by:

$$
\begin{aligned}
& \left.\begin{array}{l}
\phi_{q}^{R}\left(x^{ \pm}, y, z\right) \\
\phi_{q}^{R}\left(x, \pm \frac{w}{2}, z\right)
\end{array}\right\}=\sum_{n=0}^{\infty} \sum_{m=0}^{M} Z_{n}(z) \theta_{q}\left\{\begin{array}{l}
P_{m}(y) \alpha_{n m, q}^{R \pm}, \\
P_{m}(x) \beta_{n m, q}^{R_{ \pm}},
\end{array},\right. \\
& \left.\begin{array}{l}
\phi^{S}\left(x^{ \pm}, y, z\right) \\
\phi^{S}\left(x, \pm \frac{w}{2}, z\right)
\end{array}\right\}=\sum_{m=0}^{M} Z_{0}(z)\left\{\begin{array}{l}
P_{m}(y) \alpha_{0 m}^{S_{ \pm}} \\
P_{m}(x) \beta_{0 m}^{S_{ \pm}},
\end{array}\right. \\
& x \in(-b+L, b+L), \quad y \in\left(-\frac{w}{2}, \frac{w}{2}\right) .
\end{aligned}
$$

\section{References}

Adamo, A., Mei, C.C., 2005. Linear response of Venice storm gates to incident waves. Proc. R. Soc. A 461, 1711-1734.

Babarit, A., Hals, J., Muliawan, M.J., Kurniawan, A., Moan, T., Krokstad, J., 2012. Numerical benchmarking study of a selection of wave energy converters. Renew. Energy 41, 44-63.

Eriksson, M., Isberg, J., Leijon, M., 2005. Hydrodynamic modelling of a direct drive wave energy converter. Int. J. Eng. Sci. 43, 1377-1387.

Evans, D., 1988. The maximum efficiency of wave-energy devices near coast lines. 
Appl. Ocean Res. 10, 162-164.

Evans, D., Porter, R., 1995. Hydrodynamic characteristics of an oscillating water column device. Appl. Ocean Res. 17, 155-164.

Evans, D., Porter, R., 1996. Hydrodynamic characteristics of a thin rolling plate in finite depth of water. Appl. Ocean Res. 18, 215-228.

Falnes, J., Hals, J., 2012. Heaving buoys, point absorbers and arrays. Philos. Trans. R. Soc. A 370, 246-377.

Goda, Y., 2012. Random Seas and Design of Maritime Structures, 3rd edition. World Scientific, Singapore.

Gradshteyn, I.S., Ryzhik, I.M., 2007. Tables of Integrals Series and Products. Academic Press, San Diego.

Hasselmann, K., Barnett, T.P., Bouws, E., Carlson, H., Cartwright, D.E., Enke, K., Ewing. J.A., Gienapp, K., Hasselmann, D.E., Kruseman, P., Meerburg, A., Muller, P., Olbers, D.J., Richter, K., Sell, W., Walden, H., 1973. Measurements of wind-wave growth and swell decay during the Joint North Sea Wave Project (JONSWAP). Dtsch. Hydrogr. Zeit. R. A 8 (12) 95pp.

Kaya, A.C., Erdogan, F., 1987. On the solution of integral equations with strongly singular kernels. Q. Appl. Math. 45, 105-122.

Li, G., Mei, C.C., 2003. Natural modes of mobile flood gates. Appl. Ocean Res. 25, 115-126.

Linton, C.M., McIver, P., 2001. Mathematical Techniques for Wave/Structure Interactions. Chapman \& Hall/CRC.

Lovas, S., Mei, C.C., Liu, Y., 2010. Oscillating water column at a coastal corner for wave power extraction. Appl. Ocean Res. 32, 267-283.

Martins-Rivas, H., Mei, C.C., 2009. Wave power extraction from an oscillating water column along a straight coast. Ocean Eng. 36, 426-433.

McIver, P., Evans, D., 1984. The occurrence of negative added mass in free surface problems involving submerged oscillating bodies. J. Eng. Math. 18, 7-22.

Mei, C.C., Stiassnie, M., Yue, D.K.-P., 2005. Theory and Applications of Ocean Surface Waves. World Scientific, Singapore.

Michele, S., Sammarco, P., d'Errico, M., Renzi, E., Abdolali, A., Bellotti, G., Dias, F., 2015. Flap gate farm: from Venice lagoon defense to resonating wave energy production. Part2: Synchronous response to incident waves in open sea. Appl. Ocean Res. 52, 43-61.

Morse, P.M., Feshbach, H., 1981. Methods of Theoretical Physics. Parts I and II. Feshbach Publishing, New York.

Panizzo, A., Sammarco, P., Bellotti, G., De Girolamo, P., 2006. EOF analysis of complex response of venice mobile gates. J. Waterw. Port Coast. Ocean Eng. 132,
$172-179$.

Parsons, N.F., Martin, P.A., 1992. Scattering of water waves by submerged plates using hypersingular integral equations. Appl. Ocean Res. 14, 313-321.

Renzi, E., Dias, F., 2012. Resonant behaviour of an oscillating wave energy converter in a channel. J. Fluid Mech. 701, 482-510.

Renzi, E., Dias, F., 2013. Hydrodynamics of the oscillating wave surge converter in the open ocean. Eur. J. Mech. B/Fluids 41, 1-10.

Renzi, E., Doherty, K., Henry, H., Dias, F., 2014. How does Oyster work? The simple interpretation of Oyster mathematics. Eur. J. Mech. B/Fluids 47, 124-131.

Renzi, E., Abdolali, A., Bellotti, G., Dias, F., 2014. Wave power absorption from a finite array of oscillating wave surge converters. Renew. Energy 63, 55-68.

Renzi, E., Dias, F., 2014. Motion resonant modes of large articulated damped oscillators in waves. J. Fluid Struct. 49, 705-715.

Sammarco, P., Tran, H., Mei, C.C., 1997. Subharmonic resonance of Venice storm gates in waves. I: evolution equation and uniform incident waves. J. Fluid Mech. 349, 295-325.

Sammarco, P., Tran, H., Gottlieb, O., Mei, C.C., 1997. Subharmonic resonance of Venice storm gates in waves. II: sinusoidally modulated incident waves. J. Fluid Mech. 349, 327-359.

Sammarco, P., Michele, S., d'Errico, M., 2013. Flap gate farm: from Venice lagoon defense to resonating wave energy production. Part 1: natural modes. Appl. Ocean Res. 43, 206-213.

Sarkar, D., Renzi, E., Dias, F., 2013. Wave power extraction by an oscillating wave surge converter in random seas. In: Proceedings of the ASME 2013 32th International Conference on Ocean, Offshore and Arctic Engineering OMAE2013, Nantes, France.

Sarkar, D., Renzi, E., Dias, F., 2014. Wave farm modelling of oscillating wave surge converters. Proc. R. Soc. London A 470, 20140118.

Sarkar, D., Renzi, E., Dias, F., 2015. Effect of a straight coast on the hydrodynamics and performance of the Oscillating Wave Surge Converter. Ocean Eng. 105, 25-32.

Srokosz, M.A., Evans, D.V., 1979. A theory for wave-power absorption by two independently oscillating bodies. J. Fluid Mech. 90, 337-362.

Thomas, G.P., Evans, D.V., 1979. Arrays of three-dimensional wave-energy absorbers. J. Fluid Mech. 108, 67-88.

Whittaker, T., Folley, M., 2012. A nearshore oscillating wave surge converters and the development of Oyster. Philos. Trans. R. Soc. A 370, 345-364. 